\title{
Green-nanochemistry for safe environment: bio-friendly synthesis of fluorescent monometallic (Ag and Au) and bimetallic (Ag/Au alloy) nanoparticles having pesticide sensing activity
}

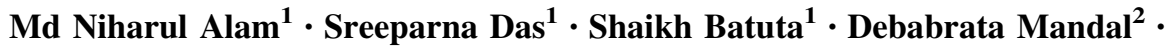 \\ Naznin Ara Begum ${ }^{1}$
}

Received: 19 August 2016/Accepted: 1 November 2016/Published online: 14 November 2016

(C) The Author(s) 2016. This article is published with open access at Springerlink.com

\begin{abstract}
Aqueous methanol (water:methanol 20:80) extract of leaves (AMEL) of Indian curry leaf plant was found to be highly efficient in the rapid and controlled synthesis of stable and fluorescent monometallic (Ag and $\mathrm{Au})$ and also bimetallic (Ag/Au alloy) nanoparticles with wide spectrum of task specific morphologies under sonochemical condition. The nanoparticles synthesized by the present economically viable and environment-friendly protocol showed characteristic fluorescence activity. This was exploited in the fluorometric sensing of the dithiocarbamate pesticide, Mancozeb in aqueous medium. The surface chemistry of these nanoparticles was extensively studied to understand their sensing activity. The naturally occurring flurophoric/chromophoric compounds (carbazole alkaloids and polyhydroxy flavonoid) present in AMEL instilled (in situ) strong and characteristic fluorescent behavior to the synthesized nanoparticles which opened up their utility as the fluorometric sensors and detectors for pesticides in aqueous medium.
\end{abstract}

Keywords Monometallic (Ag and $\mathrm{Au}$ ) nanoparticles . Bimetallic (Ag/Au alloy) nanoparticles · Indian curry leaf

Electronic supplementary material The online version of this article (doi:10.1007/s40097-016-0209-y) contains supplementary material, which is available to authorized users.

Naznin Ara Begum

naznin.begum@visva-bharati.ac.in

1 Department of Chemistry, Visva-Bharati (Central University), Santiniketan 731 235, India

2 Department of Chemistry, University College of Science and Technology, University of Calcutta, 92, Acharya Prafulla Chandra Road, Kolkata 700 009, India plant $\cdot$ Fluorometric sensing $\cdot$ Mancozeb $\cdot$ Dithiocarbamate pesticides

\section{Introduction}

Energy, environment, and human health have emerged as the main concerns not only in the research arena, but also in all aspects of our lives. In this connection, nanomaterials (e.g., metal nanoparticles) with their unique structure-dependent properties are emerging as a good promise in offering solutions in each of these priority areas. Metal nanoparticles (NPs) are being explored enormously in recent time because of their distinctive catalytic, electronic, optical, and structural properties. Subsequently, these NPs are being explored extensively to develop novel catalysts, sensors/biosensors, nanoelectronic devices, and medical diagnostic tools. The usefulness of these NPs depends critically on their morphology, composition (alloy or core-shell), and surface structure [1-3]. Thus, the design and development of simple, but energy-efficient, economic, and eco-friendly synthetic protocols for metal NPs with tailor-made structures, capable of serving specific task and biocompatibility, are the highly cherished goals for the researchers working in the field of nanoscience and nanotechnology.

Though a vast number of chemical and physical methods of synthesis are available for metal NPs, these methods are not free from drawbacks [4-6]. Sometimes, the reactants, precursors, and solvents used in the chemical synthetic methods are found to be toxic and potentially hazardous [4, 5]. Formation of toxic by-product is another problem associated with these methods [4-6]. On the other hand, in the case of physical method of synthesis of metal NPs, high temperature and pressure are required leading to the vast consumption of energy [7]. 
Therefore, researchers working in the field of nanochemistry are in an incessant quest for the new and alternative synthetic routes which are more dexterous, economic, hazard free, and environmentally viable and can yield metal NPs with desirable structural activities. In this context, an alternative synthetic strategy is being developed in the recent time which is based upon the principles of Green Chemistry [8-10]. Very often, these green chemical synthetic protocols (applicable at room temperature, pressure, and in very simple laboratory setup) take advantage of non-toxic, green multifunctional agents (GMAs) derived from the biological sources ranging from unicellular organisms to higher plants [6, 8-15].

In our previous studies, we have observed that the plant extract is very unique as GMA, because it is the source of wide spectrum of bioactive natural products which not only actively take part in the NP synthesis process, control the morphologies and surface structure/chemistry of the Ag and Au NPs synthesized by the GMA, but also impose their chromophoric/fluorophoric behavior to the synthesized NPs to make them either photoactive or fluorescent $[6,16,17]$. Recently, there is a remarkable rise of the use of the fluorescent metal NPs for sensing/biosensing process $[18,19]$. These are very much significant in the development of disease (cancer) diagnostic tools and tumor biomarkers $[18,19]$. On the other hand, metal NPs having characteristic fluorophoric/chromophoric activities also have immense applications in the colorimetric and fluorometric sensing of pesticides [19-22]. This opened up a new direction towards the synthesis of metal NPs having surfaces functionalized with fluorescent molecules or appropriate sensing molecular moieties [19-22]. However, in most of the cases, this type of metal NPs is synthesized by the conventional chemical method, and mostly, synthetic fluorophores have been used as adsorbates [19-22].

India is a country of diverse range of medicinal and aromatic plants which are the integral part of Indian traditional medicine. One of such plants is Indian curry leaf plant (Murraya koenigii Spreng.; Family: Rutaceae) and it is non-toxic and less expensive, therefore, easily available. Leaves of this traditionally used medicinal plant are extensively used in Indian cuisine, and as a whole, this plant is the rich sources of wide spectrum of multifunctional and biologically active natural products [23, 24].

In the present paper, we have demonstrated the excellent efficacy of the aqueous methanol (hydro-alcohol solvent) (water:methanol 20:80) extract of leaves (AMEL) of Indian curry leaf plant along with its active components, e.g., koenigine (A), koenidine (B), girinimbine (C), mahanimbine (D), and quercetin (Fig. 1) in the rapid and controlled synthesis of stable monometallic Ag and Au NPs and also $\mathrm{Ag} / \mathrm{Au}$ bimetallic alloy NPs under sonochemical condition. Our method is based on a non-toxic, very cheap, and widely abundant GMA, i.e., leaves of Indian curry leaf plant are edible. Moreover, collection of leaves did not destruct the tree. At the same time, this protocol can be performed at room temperature and pressure using a very easy lab setup. There is also not any possibility of the formation of toxic by-products. Thus, it is simple, economic, and environment friendly.

These synthesized NPs showed characteristic fluorescence activity. Exploring the applicability of the NPs synthesized by plant-based GMA and having tailored structural properties is essential to assess the usefulness of an NP synthesis protocol. In the present case, we have found that AMEL itself along with its active chemical constituents (which have the characteristic fluorescent activity) controlled the surface chemistry of the synthesized NPs and also imposed specific fluorescent behavior (in situ) to them which opened up their possible utility as the eco-friendly and easily synthesizable fluorometric sensors and detectors for hazardous dithiocarbamate pesticides/fungicide, such as Mancozeb (Fig. 1), which are extensively used in agriculture industries in aqueous medium [21, 22].

\section{Experimental section}

\section{Materials}

Chloroauric acid $\left(\mathrm{HAuCl}_{4}\right)$ and silver nitrate $\left(\mathrm{AgNO}_{3}\right)$ (Sigma Aldrich) were used as the sources of $\mathrm{Au}^{3+}$ and $\mathrm{Ag}^{+}$ ions, respectively. Rest of the chemicals used for the present work was of analytical grade. All analyses were done in Milli-Q (Milli-Q Academic with $0.22 \mathrm{~mm}$ Millipak R-40) water. Mancozeb was obtained from Indofil Industries Ltd., India.

\section{Instrumentation}

The formation and growth of the NPs were examined with the help of UV-Vis spectroscopy. Absorption spectra of the sample solutions were recorded on Perkin Elmer Lambda 35 spectrophotometer, where as fluorescence spectra of all the experimental solutions were recorded on a Perkin Elmer LS55 fluorimeter. All spectroscopic measurements were done at $25^{\circ} \mathrm{C}$ with an excitation wavelength $\left(\lambda_{\mathrm{ex}}\right)$ of $308 \mathrm{~nm}$ to obtain maximum fluorescence intensity. The spectrum of each of the experimental solutions remained unchanged for sufficiently long periods of time during which the spectroscopic experiments were finished. Therefore, the possibility of the decomposition of any of the experimental solutions which could change the spectroscopic results can be safely ruled out. 
Fig. 1 Chemical structures of koenigine (A), koenidine (B), girinimbine $(\mathbf{C})$, mahanimbine (D), quercetin and Mancozeb

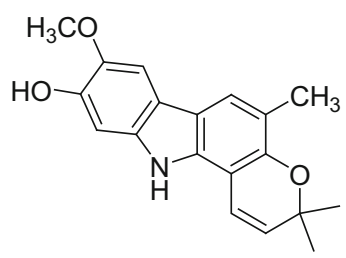

(A)

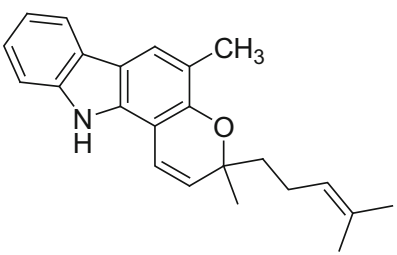

(D)<smiles>COc1cc2[nH]c3c4c(c(C)cc3c2cc1OC)OC(C)(C)C=C4</smiles>

(B)<smiles>O=c1c(O)c(-c2ccc(O)c(O)c2)oc2cc(O)cc(O)c12</smiles>

Quercetin<smiles>Cc1cc2c([nH]c3ccccc32)c2c1OC(C)(C)C=C2</smiles>

(C)<smiles>S=C([S-])NCCNC(=S)[S-]</smiles>

Mancozeb
Thermal responses of the NPs synthesized by the present protocol were estimated by Thermo gravimetric analysis (TGA). TGA of NP samples was done using a Pyris Diamond TG/DTA (Perkin Elmer, STA-6000) thermal analyzer. The experiment was set in the temperature range of $40-900{ }^{\circ} \mathrm{C}$ and at a heating rate of $15^{\circ} \mathrm{C} \mathrm{min}{ }^{-1}$ under nitrogen atmosphere.

Fourier-transformation-infrared (FT-IR) spectra of the experimental samples were recorded on a Shimadzu FTIR8400S PC instrument. Prior to FT-IR measurements, NP solutions were centrifuged at $14,000 \mathrm{rpm}$ for $30 \mathrm{~min}$ followed by their drying in vacuum.

Melting points of the isolated compounds (A, B, C, and D) were determined by an electro-thermal apparatus and were uncorrected. All the compounds were purified to A. R. grade before NP synthesis. Purity of the compounds was routinely tested with the help of thin layer chromatography (TLC). TLC studies of these compounds were carried out in silica gel GF 254 pre-coated plates. The structures of the isolated compounds were elucidated and confirmed by comparing their melting point, IR, and ${ }^{1} \mathrm{H}$ NMR data. ${ }^{1} \mathrm{H}$ NMR spectroscopy was recorded at $400 \mathrm{MHz}$ in a Brucker Avance-400 spectrometer in $\mathrm{DMSO}-\mathrm{d}_{6}$ or $\mathrm{CDCl}_{3}$ solution.

Shape and size of the synthesized particles were studied by transmission electron microscopy (TEM). For the preparation of samples for TEM, the NP solution was dropcoated onto the carbon-coated copper grids of size 400 mesh. The films on the grids were dried prior to the TEM measurement by a JEOL JEM-2100 instrument.

For further characterization of the synthesized NPs, powder X-ray diffraction (XRD) analysis was done. For the preparation of XRD samples, NP solutions were centrifuged at 14,500 rpm for $30 \mathrm{~min}$ and the supernatant was discarded. Then, NPs were dispersed in water and vortexed. After repeating these steps for three times, the residue part was dried in vacuum. The dry powder obtained was spreaded evenly on a quartz slide to perform the XRD studies. The XRD patterns were recorded using the Rigaku Ultima IV diffractometer attached with D/tex ultra detector and $\mathrm{CuK} \alpha$ source operating at $50 \mathrm{~mA}$ and $40 \mathrm{kV}$. The scan range was fixed at $2 \theta=25^{\circ}-85^{\circ}$ with a stepwise size of $0.01^{\circ}$.

Zeta-potential measurement of the experimental solutions was done by Malvern Zetasizer Nano ZS-ZEN 3600 (Malvern Instruments Ltd, UK) instrument. Disposable cuvettes ( $1 \mathrm{~mL}$ volume) specific for this instrument were used for this purpose. Prior to the experiment, all NP samples were diluted appropriately with Milli-Q water to observe optimum signal intensity. Five replications were done for each sample.

Collection of leaves of Indian curry leaf plant, the extraction procedure to get aqueous methanol extract (AMEL) of dried and pulverized leaves, and isolation and identification of the active chemical constituents of AMEL are discussed in detail in the Electronic Supplementary Material (page S2).

This gummy mass obtained from AMEL (GAMEL) obtained after evaporation of the solvent under reduced pressure was stored at $4{ }^{\circ} \mathrm{C}$ and diluted appropriately in Milli-Q water by sonication before its use as green multifunctional agent (GMA) in the synthesis of three types of NPs: monometallic Ag, $\mathrm{Au}$, and bimetallic Ag/Au NPs (discussed in later section). We have quantified the total flavonoid and polyphenol contents of AMEL using the standard colorimetric methods $[25,26]$ which are found to be $102.5 \mathrm{mg}$ quercetin equivalent $\mathrm{g}^{-1}$ and $214.3 \mathrm{mg}$ gallic acid equivalent $\mathrm{g}^{-1}$. Detailed procedures are discussed in the Electronic Supplementary Material (page S3). In our previous work, we have observed that leaves of Indian curry leaf plants are rich in polyphenols, flavonoids, such as quercetin, and quercetin-3-glucoside [16]. Other reported flavonoids present in aqueous methanol extract of 
leaves of this plant are myricetin-3-galactoside, quercetin$O$-pentohexoside, quercetin-3-diglucoside, quercetin-3-Orutinoside, quercetin-3-acetylhexoside, kaempferol- $O$-glucoside, and kaempferol-aglucoside [24].

We have also isolated and identified four carbazole alkaloids by column chromatography of the gummy mass obtained from AMEL. Detailed isolation procedure has been given in the Electronic Supplementary Material (page S3). These isolated compounds are koenigine (A), koenidine (B), girinimbine (C), and mahanimbine (D) (Fig. 1).

\section{Method of synthesis of monometallic (Ag and Au) and bimetallic (Ag/Au) NPs by AMEL and its chemical constituents (A, B, C, D, and quercetin) as GMA}

In general, the synthesis of Ag NP was initiated by adding $200 \mu \mathrm{L}$ of $0.1(\mathrm{M})$ aqueous solution of $\mathrm{AgNO}_{3}$ to $10 \mathrm{~mL}$ of aqueous solution of GAMEL $\left(400 \mu \mathrm{g} \mathrm{mL}^{-1}\right)$ in a $100 \mathrm{~mL}$ conical flask. The final concentration of $\mathrm{Ag}^{+}$ions in the reaction mixture was maintained at $2 \times 10^{-3}(\mathrm{M})$. The $\mathrm{pH}$ of the mixture was adjusted at 9 (optimum $\mathrm{pH}$ for the reaction) by adding dilute aqueous solution of $\mathrm{NaOH}$. A series of trial experiments were run at several lower and higher $\mathrm{pH}$ ranges to get an idea about the optimum $\mathrm{pH}$ for this reaction. The conical flask containing the reaction mixture was placed in an ultrasonic bath (Branson 1510), and the reaction mixture was sonicated at $40 \mathrm{kHz}$ at room temperature. The reaction mixture turned to golden yellow color within a minute indicating the onset of formation of Ag NPs. The progress of the reaction was followed by monitoring the absorbance of the reaction mixture at regular interval of times. The absorption peak is assigned to the surface plasmon resonance (SPR) band of Ag NP formed by the reduction of $\mathrm{Ag}^{+}$ions by GMA.

We have followed similar method for the synthesis of $\mathrm{Au}$ NPs except in this time, and we have added $200 \mu \mathrm{L}$ of 0.1 (M) aqueous $\mathrm{HAuCl}_{4}$ solution instead of $\mathrm{AgNO}_{3}$ aqueous solution. In this case, the final concentration of $\mathrm{Au}^{3+}$ ions was $2 \times 10^{-3}(\mathrm{M})$. Within $2 \mathrm{~min}$, a pink coloration was observed indicating the onset formation of $\mathrm{Au}$ NPs.

For the synthesis of bimetallic $\mathrm{Ag} / \mathrm{Au} \mathrm{NPs}$, different amounts of $0.1(\mathrm{M})$ aqueous $\mathrm{AgNO}_{3}$ and $0.1(\mathrm{M})$ aqueous $\mathrm{HAuCl}_{4}$ solutions were added to $10 \mathrm{~mL}$ of aqueous solution of GAMEL $\left(400 \mu \mathrm{g} \mathrm{mL}^{-1}\right)$, while other reaction parameters were kept unaltered (Table 1). In each case, characteristic change of the color of reaction mixture was observed within 2 min which was the primary indication of the formation of bimetallic alloy NPs.

In the following section, we have discussed the method of synthesis of the NPs by individual chemical constituents of AMEL isolated by us.
In each case, the stock solution of each of the compounds $(\mathbf{A}) /(\mathbf{B}) /(\mathbf{C}) /(\mathbf{D})$ was prepared by dissolving $2 \mathrm{mg}$ of the compound in $3 \mathrm{~mL}$ of ethanol. For Ag NP synthesis by (A), $50 \mu \mathrm{L}$ of its stock solution was added to $5 \mathrm{~mL}$ of $10 \mathrm{mM}$ SDS solution (to avoid the precipitation of the organic compounds in aqueous medium) and the addition of aqueous $\mathrm{NaOH}$ solution was done to adjust the $\mathrm{pH}$ at 9 (optimum pH). $50 \mu \mathrm{L}$ of $0.05(\mathrm{M})$ aqueous solution of $\mathrm{AgNO}_{3}$ was added to this reaction mixture, so that the final $\mathrm{Ag}^{+}$ion concentration became $0.5 \times 10^{-3}(\mathrm{M})$. This reaction mixture was sonicated at $40 \mathrm{kHz}$ at room temperature, and golden yellow coloration was developed within 5 min indicating the onset of formation of Ag NP. Similar method was found to be useful for the synthesis of Ag NPs either by $(\mathbf{B}) /(\mathbf{C})$ or $(\mathbf{D})$.

The compounds (A), (B), (C), and (D) were also found to be efficient in the synthesis of Au NPs. Similar method has been followed for this synthesis except that $50 \mu \mathrm{L}$ of $0.05(\mathrm{M})$ aqueous $\mathrm{HAuCl}_{4}$ solution was used instead of $\mathrm{AgNO}_{3}$ aqueous solution. In this case, the final concentration of $\mathrm{Au}^{3+}$ ions became $0.5 \times 10^{-3}(\mathrm{M})$. Within 5 min of sonication, a blue coloration was observed which indicated the onset formation of Au NPs.

Similarly, for bimetallic Ag/Au NPs (1:1) synthesis by the compound $(\mathbf{A}) /(\mathbf{B}) /(\mathbf{C}) /(\mathbf{D}), 50 \mu \mathrm{L}$ of the stock solution of the respective compound was added to $5 \mathrm{~mL}$ of $10 \mathrm{mM}$ SDS solution followed by simultaneous addition of $25 \mu \mathrm{L}$ of $0.05 \mathrm{M}$ aqueous $\mathrm{AgNO}_{3}$ and $25 \mu \mathrm{L}$ of $0.05 \mathrm{M}$ aqueous $\mathrm{HAuCl}_{4}$ solution keeping other parameters unaltered (Table 2). Color generation was observed within 5 min which indicated the bimetallic alloy formation.

We have also shown the efficacy of quercetin, abundantly found polyhydroxy flavonoid in leaves of Indian Curry leaf plant towards the synthesis of $\mathrm{Au}, \mathrm{Ag}$, and $\mathrm{Ag}$ / $\mathrm{Au}$ NPs. For Ag NP synthesis by quercetin, $100 \mu \mathrm{L}$ of 0.05 (M) aqueous solution of $\mathrm{AgNO}_{3}$ was added to the $10 \mathrm{~mL}$ aqueous solution of quercetin $\left(0.025 \mathrm{mg} \mathrm{mL}^{-1}\right)$. Aqueous $\mathrm{NaOH}$ solution was added to the reaction mixture to adjust the $\mathrm{pH}$ at 9 (optimum $\mathrm{pH}$ ). The final $\mathrm{Ag}^{+}$ion concentration became $0.5 \times 10^{-3}(\mathrm{M})$. This mixture was sonicated at $40 \mathrm{kHz}$ at room temperature, and golden yellow coloration was developed within $5 \mathrm{~min}$ indicating the onset of formation of Ag NP (Table 2). Similar method using $100 \mu \mathrm{L}$ of $0.05(\mathrm{M})$ aqueous $\mathrm{HAuCl}_{4}$ solution was followed for the synthesis of monometallic $\mathrm{Au}$ NPs. For bimetallic $\mathrm{Ag} / \mathrm{Au}$ NP (1:1) synthesis, $50 \mu \mathrm{L}$ of $0.05(\mathrm{M})$ aqueous $\mathrm{AgNO}_{3}$ and $50 \mu \mathrm{L}$ of $0.05(\mathrm{M})$ aqueous $\mathrm{HAuCl}_{4}$ solution were added simultaneously (Table 2) and sonicated as stated earlier. In these two cases, we kept all other reaction parameters unchanged. Color was developed within 5 min which indicated the formation of NPs. In all the cases, the progress of the reaction was monitored by measuring the absorbance of the reaction mixture with time. 
Table 1 Synthesis of monometallic Ag, Au, and bimetallic Ag/Au alloy NPs using AMEL as GMA

\begin{tabular}{lllllc}
\hline Synthesized NP & Set & Conc. of $\mathrm{Ag}^{+}(\mathrm{M})$ & Conc. of $\mathrm{Au}^{3+}(\mathrm{M})$ & $\begin{array}{l}\text { Position of SPR } \\
\text { band }(\mathrm{nm})\end{array}$ & $\begin{array}{l}\text { Inset of formation } \\
(\mathrm{min})\end{array}$ \\
\hline Monometallic Ag & $\mathrm{a}$ & $2 \times 10^{-3}$ & 0 & 408 & 2 \\
Bimetallic Ag/Au alloy & $\mathrm{b}$ & $1.5 \times 10^{-3}$ & $0.5 \times 10^{-3}$ & 432 & 2 \\
Bimetallic Ag/Au alloy & $\mathrm{c}$ & $1 \times 10^{-3}$ & $1 \times 10^{-3}$ & 459 & 2 \\
Bimetallic Ag/Au alloy & $\mathrm{d}$ & $0.5 \times 10^{-3}$ & $1.5 \times 10^{-3}$ & 487 & 2 \\
Monometallic Au & $\mathrm{e}$ & 0 & $2 \times 10^{-3}$ & 522 & 2 \\
\hline
\end{tabular}

Table 2 Synthesis of monometallic Ag, Au and bimetallic Ag/Au alloy NPs by individual compounds

\begin{tabular}{|c|c|c|c|c|c|c|}
\hline Synthesized NP & Set & Compound & $\begin{array}{l}\text { Conc. of } \mathrm{Ag}^{+} \\
\text {(M) }\end{array}$ & $\begin{array}{l}\text { Conc. of } \mathrm{Au}^{3+} \\
\text { (M) }\end{array}$ & $\begin{array}{l}\text { Position of SPR } \\
\text { band }(\mathrm{nm})\end{array}$ & $\begin{array}{l}\text { Inset of formation } \\
\text { (min) }\end{array}$ \\
\hline Monometallic Ag & $\mathrm{f}$ & Koenigine (A) & $0.5 \times 10^{-3}$ & 0 & 421 & 5 \\
\hline Bimetallic $\mathrm{Ag} / \mathrm{Au}$ alloy & $\mathrm{g}$ & & $0.25 \times 10^{-3}$ & $0.25 \times 10^{-3}$ & 488 & 5 \\
\hline Monometallic $\mathrm{Au}$ & $\mathrm{h}$ & & 0 & $0.5 \times 10^{-3}$ & 564 & 5 \\
\hline Monometallic Ag & $\mathrm{i}$ & Koenidine (B) & $0.5 \times 10^{-3}$ & 0 & 424 & 5 \\
\hline Bimetallic $\mathrm{Ag} / \mathrm{Au}$ alloy & $\mathrm{j}$ & & $0.25 \times 10^{-3}$ & $0.25 \times 10^{-3}$ & 482 & 5 \\
\hline Monometallic $\mathrm{Au}$ & $\mathrm{k}$ & & 0 & $0.5 \times 10^{-3}$ & 565 & 5 \\
\hline Monometallic Ag & 1 & Girinimbine $(\mathbf{C})$ & $0.5 \times 10^{-3}$ & 0 & 426 & 5 \\
\hline Bimetallic $\mathrm{Ag} / \mathrm{Au}$ alloy & $\mathrm{m}$ & & $0.25 \times 10^{-3}$ & $0.25 \times 10^{-3}$ & 506 & 5 \\
\hline Monometallic Au & $\mathrm{n}$ & & 0 & $0.5 \times 10^{-3}$ & 572 & 5 \\
\hline Monometallic Ag & o & Mahanimbine (D) & $0.5 \times 10^{-3}$ & 0 & 422 & 5 \\
\hline Bimetallic $\mathrm{Ag} / \mathrm{Au}$ alloy & $\mathrm{p}$ & & $0.25 \times 10^{-3}$ & $0.25 \times 10^{-3}$ & 511 & 5 \\
\hline Monometallic Au & $\mathrm{q}$ & & 0 & $0.5 \times 10^{-3}$ & 537 & 5 \\
\hline Monometallic Ag & $\mathrm{r}$ & Quercetin & $0.5 \times 10^{-3}$ & 0 & 406 & 5 \\
\hline Bimetallic $\mathrm{Ag} / \mathrm{Au}$ alloy & s & & $0.25 \times 10^{-3}$ & $0.25 \times 10^{-3}$ & 466 & 5 \\
\hline Monometallic $\mathrm{Au}$ & $\mathrm{t}$ & & 0 & $0.5 \times 10^{-3}$ & 525 & 5 \\
\hline
\end{tabular}

\section{Method of fluorometric sensing of Mancozeb, a dithiocarbamate pesticide by the synthesized NPs}

We have explored the intense fluorescence activity of the synthesized NPs solutions as a measuring tool for sensing study of the dithiocarbamate pesticide, Mancozeb in aqueous medium. We have prepared $296.11 \mu \mathrm{M}$ (100 ppm) aqueous stock solution of Mancozeb. $2.5 \mathrm{~mL}$ of the NP solution [sets a-c (Table 1); $\mathrm{f}-\mathrm{h}, \mathrm{r}-\mathrm{t}$ (Table 2)] was taken in a fluorescence cuvette followed by successive addition of different volumes (10-300 $\mu \mathrm{L})$ of stock solution of Mancozeb to it, and fluorescence spectrum was recorded after each addition. The final concentration of Mancozeb in the reaction mixture was varied from 1.18 to $31.73 \mu \mathrm{M}(0.39-10.71 \mathrm{ppm})$. The enhancement of fluorescence emission intensity or 'fluorescence turn on' of the NP solution on the addition of Mancozeb confirmed the sensing activity of the respective NP solution.

\section{Results and discussion}

\section{Formation and growth of monometallic (Ag and Au) and bimetallic (Ag/Au) NPs synthesized by AMEL and its chemical constituents as GMA}

UV-Vis spectroscopy was used to confirm the formation of the NPs by the reduction of the corresponding metal ion in aqueous solutions when exposed to the present GMA. Figure 2i(set a) shows the result of the reaction between $\mathrm{Ag}^{+}$ions and the aqueous solution of GAMEL at $\mathrm{pH} 9$. The curve denoted by broken line in Fig. $2 \mathrm{i}$ represents the absorption spectrum of aqueous solution of GMA in the absence of $\mathrm{Ag}^{+}$ions. Upon sonication of this reaction mixture at room temperature, SPR band for Ag NP appeared at $408 \mathrm{~nm}$ within $2 \mathrm{~min}$ and the intensity of this band was found to be increased with time. Finally, a saturation was observed after $7 \mathrm{~min}$ [Fig. 2ii(set a)]. Figure $2 \mathrm{i}$ (set e) shows the result of formation of the Au NPs 
when $\mathrm{Au}^{3+}$ ions were reduced by the GMA. The absorbance maxima of Au NPs formed by GMA was recorded and plotted against time, as shown in Fig. 2ii(set e). Within 2 min of the addition of $\mathrm{Au}^{3+}$ ion with continuous sonication at room temperature, the reaction mixture turned pink with the appearance of a main peak at $522 \mathrm{~nm}$.

The efficacy of the AMEL was further tested in the synthesis of three types of alloy NPs: Ag/Au (3:1), (1:1), and (1:3) NPs. $\mathrm{Ag}^{+}$and $\mathrm{Au}^{3+}$ ions present in the same solution were simultaneously reduced by the GMA to form bimetallic $\mathrm{Ag} / \mathrm{Au}$ alloy NPs which were also stabilized by the same GMA [Fig. 2i(sets b-d)]. The formation of bimetallic $\mathrm{Ag} / \mathrm{Au}$ alloy NP formation was established from the fact that the absorption spectrum showed only one plasmon band in place of two individual bands for $\mathrm{Ag}$ and Au NPs [2, 3, 5, 27, 28]. Figure 2i(sets b-d) shows the normalized absorption spectra of the reaction mixture for the simultaneous reduction of the various concentration of $\mathrm{Ag}^{+}$and $\mathrm{Au}^{3+}$ ions by AMEL (detailed concentration range is given in Table 1). For set $b$, after completion of the reduction the absorption maximum appeared at $432 \mathrm{~nm}$ and for sets $\mathrm{c}$ and $\mathrm{d}$, the corresponding maxima were observed at 459 and $487 \mathrm{~nm}$, respectively. It was observed that, only one absorbance peak was obtained for each of the bimetallic NP solutions. Moreover, in all the cases, the absorbance maxima were found to be located at the positions in between the SPR bands associated with the monometallic Ag NPs (408 nm) and Au NPs (522 nm). This is in agreement with the previously reported data $[3,27]$. Such absorption spectra cannot be observed if it was a case of simple physical mixture of monometallic $\mathrm{Ag}$ and $\mathrm{Au} \mathrm{NP}$ solutions [29]. Moreover, the spectra in Fig. 1i(sets b-d) did not have any resemblance to those exhibited by the bimetallic $\mathrm{Ag} / \mathrm{Au}$ core-shell NPs. In general, two characteristic absorption peaks are observed for the bimetallic $\mathrm{Ag} / \mathrm{Au}$ core-shell NPs [3, 27]. Moreover, as shown in Fig. 2iii, the SPR peak position, i.e., absorbance maxima of the bimetallic system, was found to be gradually red shifted linearly with the increase of concentration of $\mathrm{Au}^{3+}$ ions $(y=406.2+56.2 x)$; which further supports the formation of $\mathrm{Ag} / \mathrm{Au}$ bimetallic alloy NPs $[3,27]$. The UV-Vis absorption data thus satisfactorily confirmed the simultaneous reduction of $\mathrm{Ag}^{+}$and $\mathrm{Au}^{3+}$ ions by GMA in aqueous medium to produce the homogeneous bimetallic alloy NPs. This was further confirmed by the TEM images (discussed in later section).

\section{Formation and growth of monometallic ( $\mathrm{Ag}$ and $\mathrm{Au})$ and bimetallic (Ag/Au) NPs by isolated compounds} $(\mathrm{A}, \mathrm{B}, \mathrm{C}$, and $\mathrm{D})$ and quercetin

Plant extract is the concoction of several types of chemical compounds (natural products) which are the characteristics of a specific plant genus. These chemical constituents impart characteristic redox and stabilizing/capping activities to the plant extract making it a green multifunctional agent (GMA) in the field of synthesis and stabilization of NPs $[5,14]$. Use of plant-based GMA is being explored extensively now-a-days. However, one of the major problems associated with these types of methods is lack of fine tuning of the NP morphology and reproducibility which are essential for practical implementation of any synthetic protocol. From our previous work [3, 4, 6, 16, 27], we have observed that nature and content of these chemical constituents are major controlling parameters for these types of protocols. Therefore, identification of these compounds is very much necessary for properly scaling up of these synthetic protocols.

In our previous work, we have observed that aqueous extract of Indian curry leaf plant is rich in polyphenols and flavonoids [16]. However, in the present case, we have observed that in addition to polyphenols and flavonoids, AMEL is also rich in carbazole alkaloids, such as koenigine (A), koenidine (B), girinimbine (C), and mahanimbine (D).

Therefore, for the first time, we have explored the carbazole-based secondary metabolites of plant in the rapid synthesis of stable monometallic $\mathrm{Ag}$, $\mathrm{Au}$, and bimetallic $\mathrm{Ag} / \mathrm{Au}(1: 1) \mathrm{NPs}$.

Formation and growth of the NPs were monitored with the help of UV-Vis spectroscopy, and the results are shown in Fig. 3i-iv. The curves with broken line represent the absorption spectra of koenigine (A), koenidine (B), girinimbine (C), and mahanimbine (D), respectively, in the absence of metal ions. In all the cases, the appearance of characteristic absorption spectra clearly confirmed the formation of Ag NP, Au NP, and alloy Ag/Au (1:1) NP (Fig. 3i-iv). Position of SPR bands and the time for onset of formation of respective NPs were shown in Table 2(sets $f-q)$.

In each case of the Ag NP synthesis by the isolated compounds (A, B, C, and D), sharp SPR bands were obtained in the range of $421-426 \mathrm{~nm}$ [Fig. 3i-iv; Table 2(sets f-q)]. However, in the case of Au NPs synthesized by the same systems, much broader SPR bands (indicating the formation of diverse type NPs) were observed (Fig. 3i-iv). These were further confirmed on the basis of TEM analysis. Only one absorbance peak was observed for the formation of bimetallic alloy $\mathrm{Ag} / \mathrm{Au}$ (1:1) NPs by each of these individual compounds, and absorbance maxima were found to be located at the position intermediate to those usually observed for monometallic $\mathrm{Ag}$ and $\mathrm{Au}$ NPs. Such absorption spectra could not be obtained if simply a physical mixture of $\mathrm{Ag}$ and $\mathrm{Au}$ was formed (Fig. 3i-iv; Table 2). 


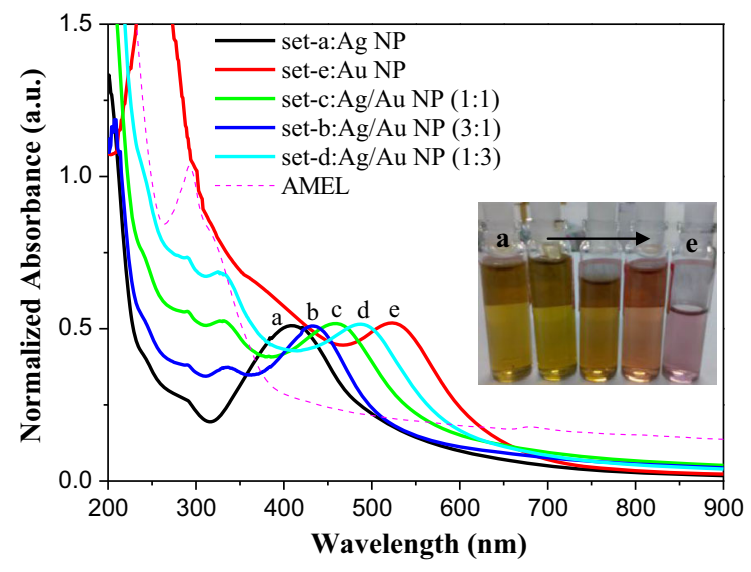

(i)

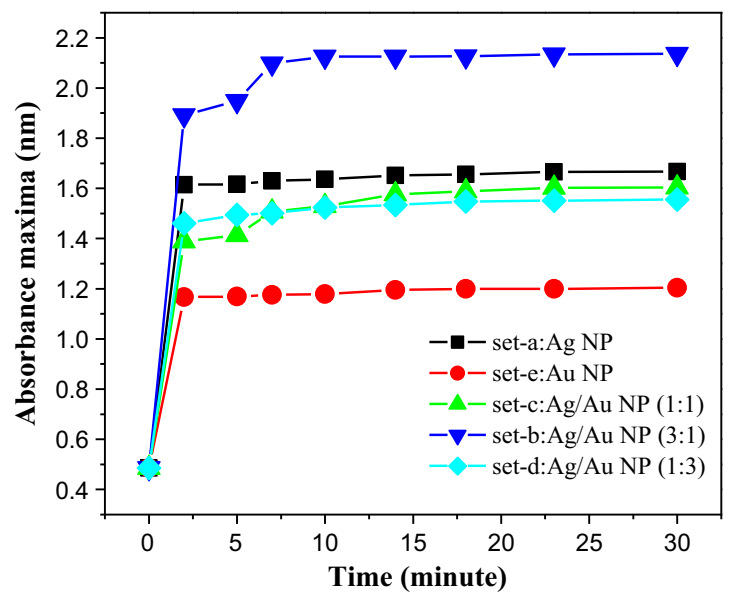

(ii)

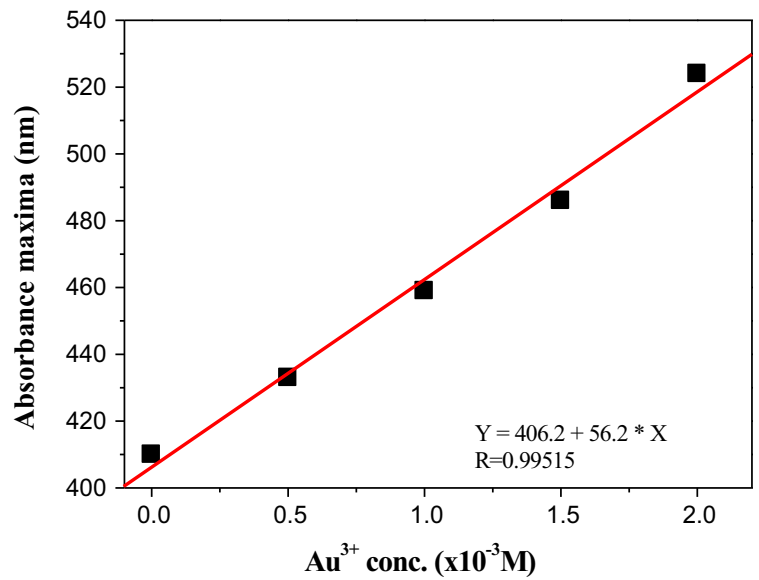

(iii)

(set-a)

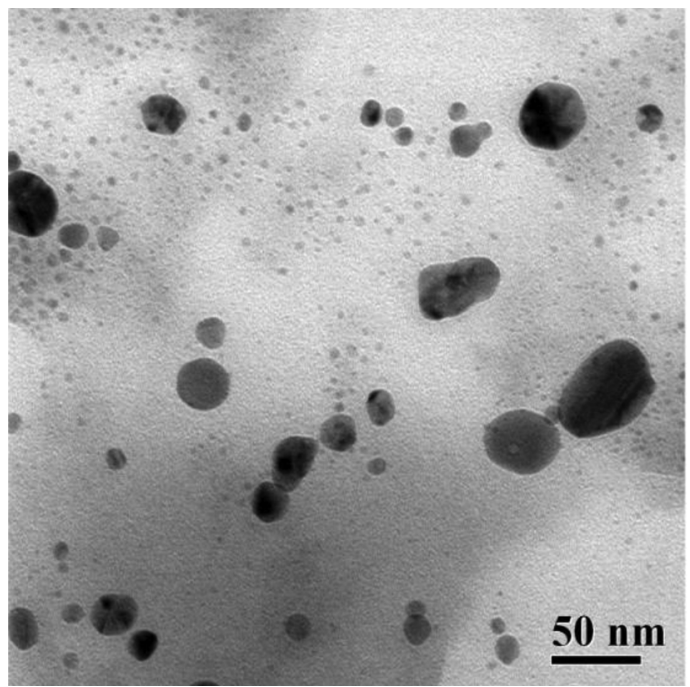

Fig. 2 i UV-visible spectra and ii change in peak absorbance with time for different sets of synthesized NPs: Ag NP (set a), Au NP (set $e$ ), and $\mathrm{Ag} / \mathrm{Au}$ alloy NP prepared at different $\mathrm{Ag} / \mathrm{Au}$ molar ratios 3:1 (set b), 1:1 (set c), and 1:3 (set d). Broken line in $\mathbf{i}$ represents absorbance of AMEL, and used as GMA. Inset shows the color of the corresponding NP solutions. iii Positions of surface plasmon

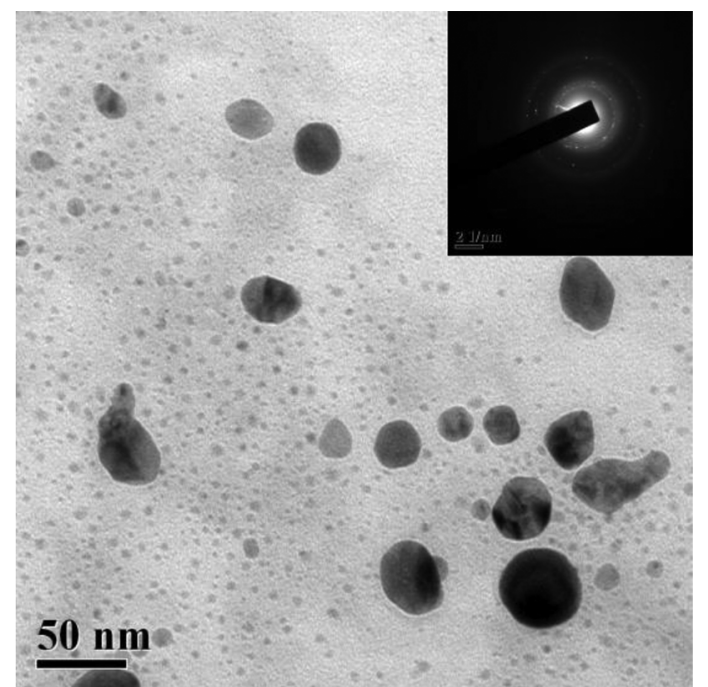

resonance band maxima was plotted as a function of the molar fraction of $\mathrm{Au}$. iv TEM images of Ag NPs (set a), Ag/Au alloy NPs prepared at different $\mathrm{Ag} / \mathrm{Au}$ molar ratios 3:1 (set b), 1:1 (set c), 1:3 (set d), and Au NPs (set e). Inset in iv shows the SAED pattern of the corresponding NPs. $\mathbf{v}$ XRD patterns and vi EDX profiles for Ag NP (set a), Au NP (set e), and Ag/Au alloy NP (1:1) (set c) 

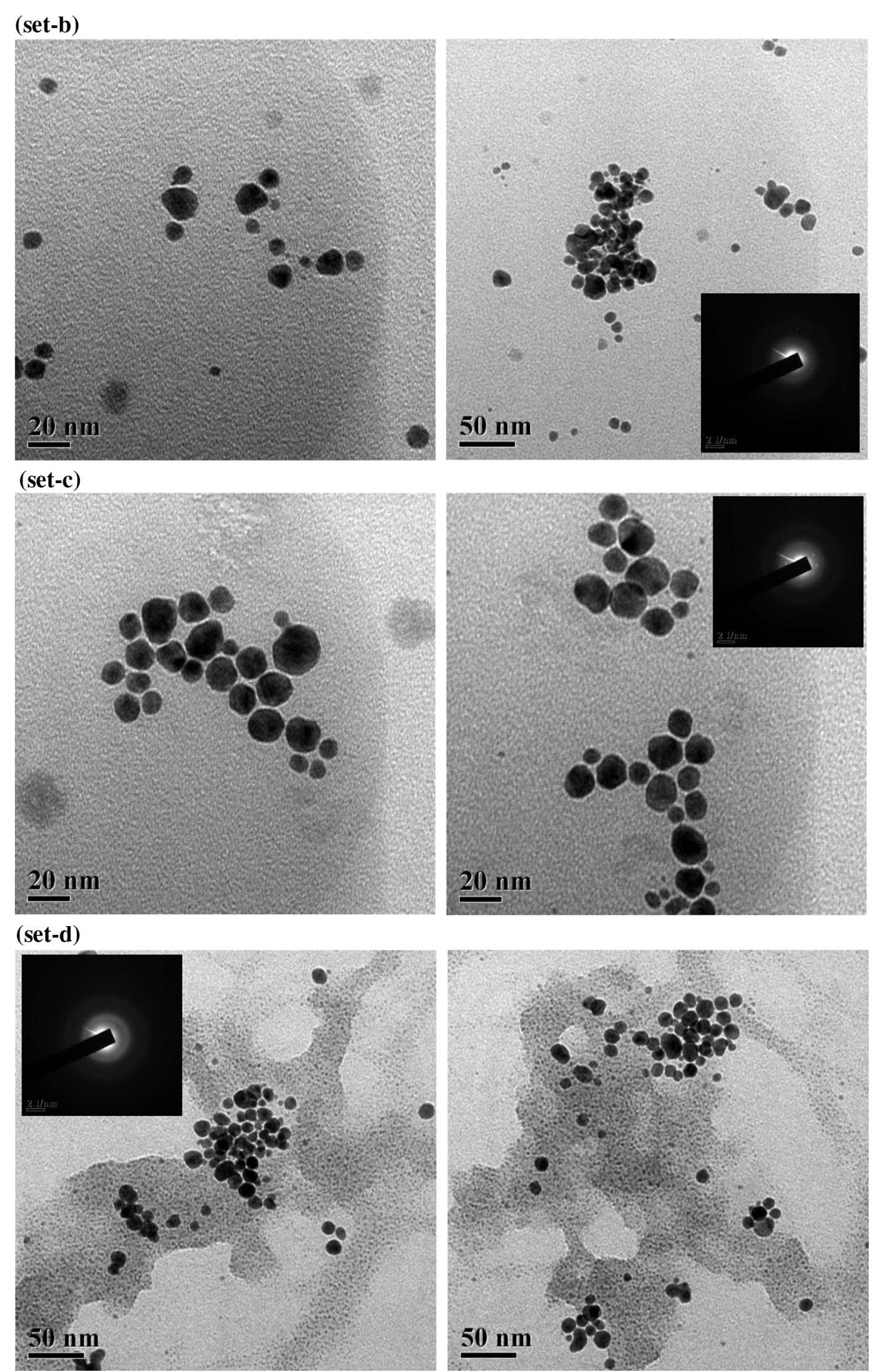

Fig. 2 continued 

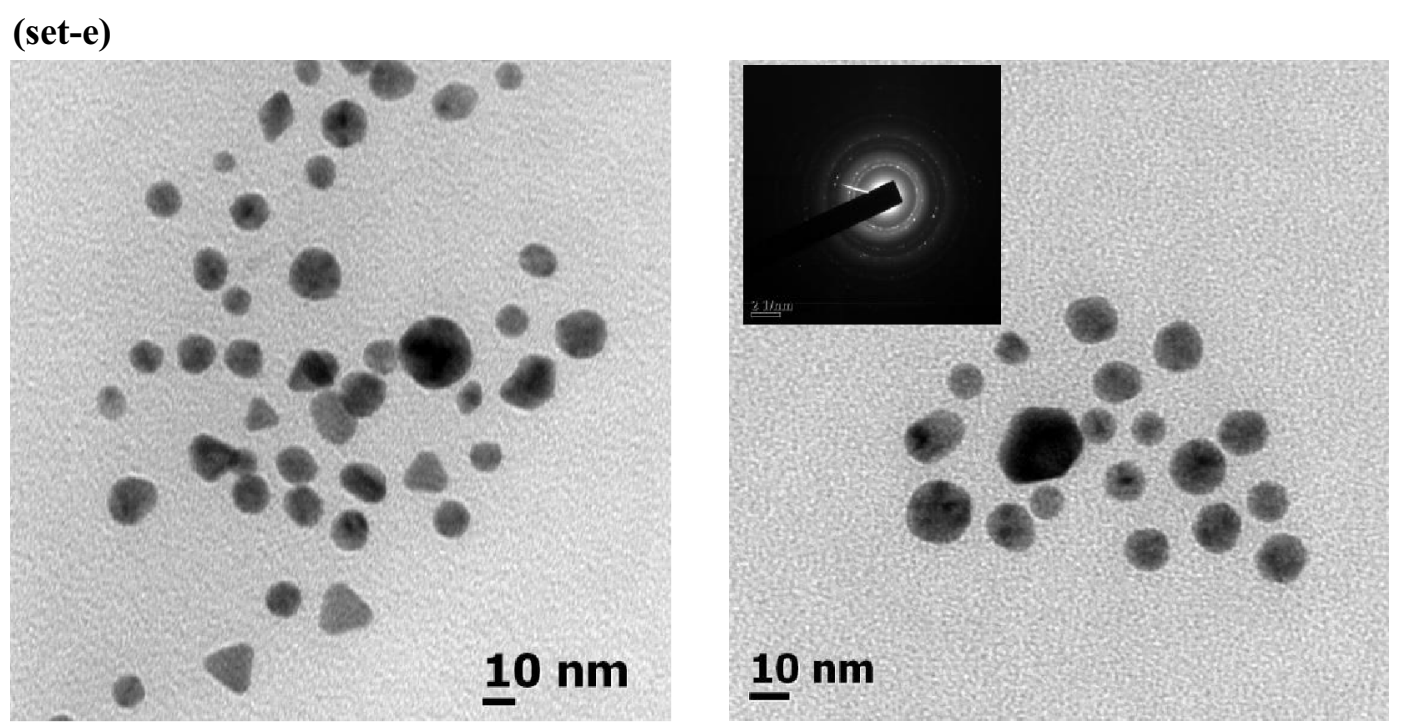

(iv)

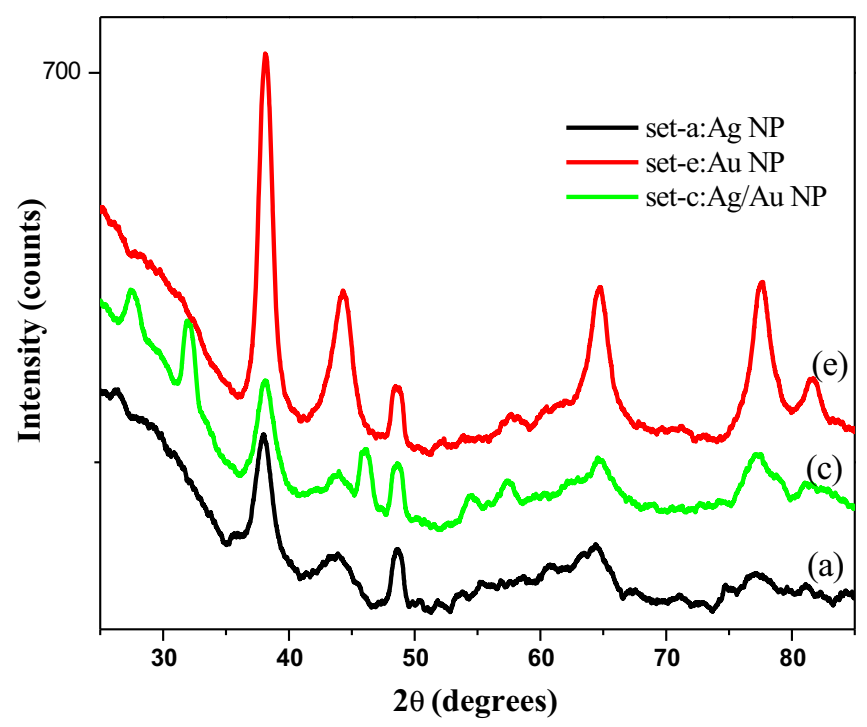

(v)

Fig. 2 continued

The same types of NPs were formed when quercetin was used as a reducing agent. Figure $3 v$ shows the UV-Vis absorption spectra of Ag NP (set r), Au NP (set t), and Ag/ Au NP (1:1) (set s) synthesized by quercetin, one of the constituents present in AMEL.

\section{Morphology of monometallic (Ag and Au)} and bimetallic (Ag/Au) NPs synthesized by AMEL and its chemical constituents as GMA

Morphology of the synthesized NPs was studied by the TEM. Figure 2iv [for sets (a) and (e)] shows the TEM images of the $\mathrm{Ag}$ and $\mathrm{Au}$ NPs synthesized by AMEL, respectively. In the case of Ag NPs, particles were found to be well separated and mostly spheroidal in shape [Fig. 2iv(set a)]. On the other hand, Au NPs of interesting morphologies, e.g., triangles and hexagons together with regular spheroidal shape NPs, were observed in the respective TEM images [Fig. 2iv(set e)].

Figure 2iv(sets b-d) shows the TEM images of the bimetallic $\mathrm{Au} / \mathrm{Ag} \mathrm{NPs}$ formed by the simultaneous reduction of $\mathrm{Ag}^{+}$and $\mathrm{Au}^{3+}$ ions in the reaction mixture having $\mathrm{Ag}^{+}: \mathrm{Au}^{3+}$ concentration ratio $3: 1$ (set b), 1:1 (set c), and 1:3 (set d). The particles formed were predominantly spherical. In the close up view, occasional aggregations are quite visible. TEM images of the bimetallic core/shell-type structure usually show electron density banding with a dark Au core and a lighter Ag 

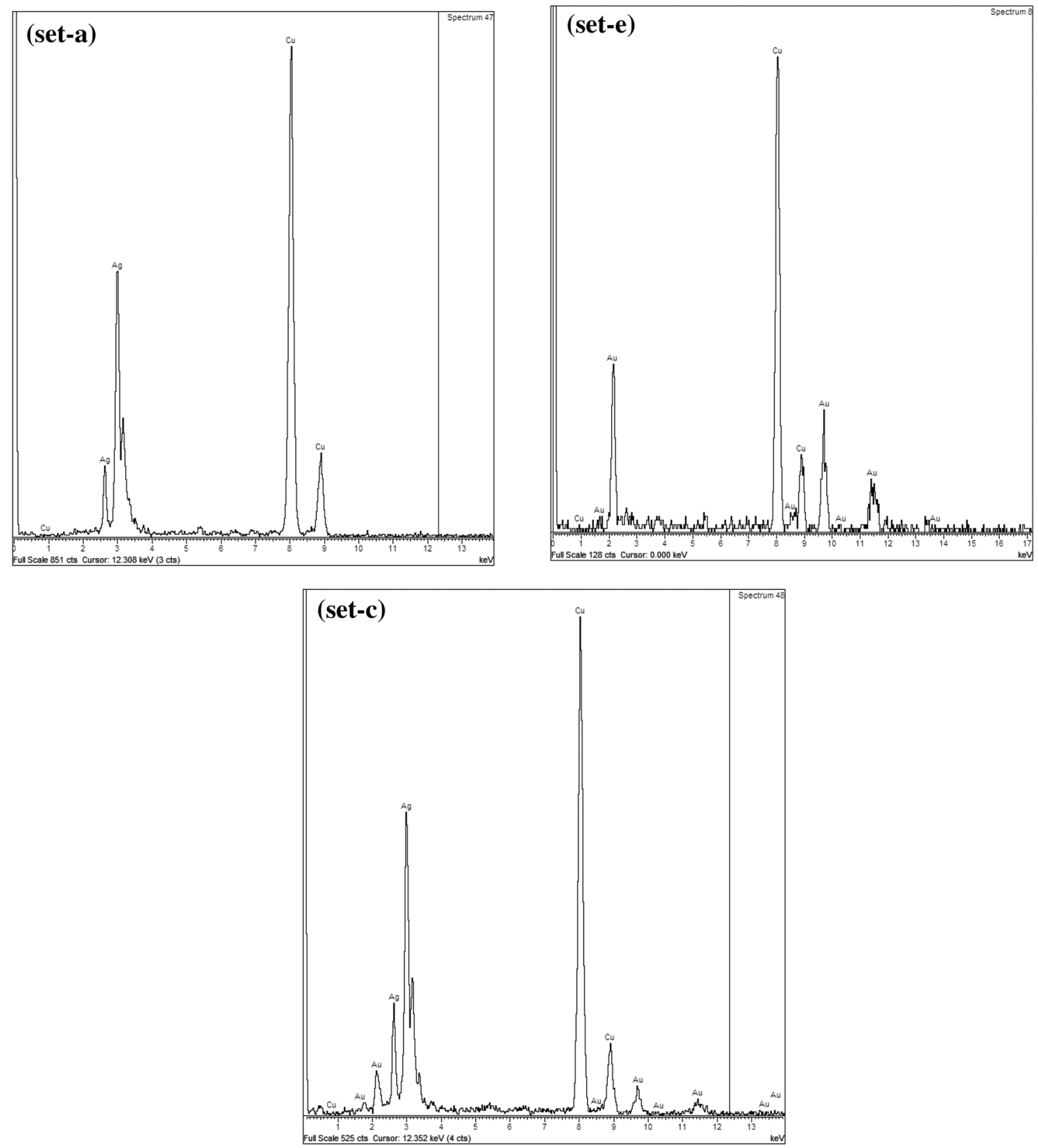

(vi)

Fig. 2 continued

shell [30] which was not observed in the present case. TEM images of the synthesized bimetallic Ag/Au (1:1) NP showed the uniform contrast for each NP. This suggested that the electron density was homogeneous within the volume of the particle [30]. Therefore, the bimetallic NPs synthesized presently were not core/shell type and these NPs closely resembled to the bimetallic alloy NPs. These results are in strong agreement with the UV-Vis spectroscopic data discussed previously and also the reported data $[3,30]$.
Compared to the mother extract (AMEL), more uniform morphologies of the NPs were observed in the case of NPs synthesized by its individual components $(\mathbf{A}, \mathbf{B}$, C, and D) (Fig. 3vi). There may be two possible reasons for this. First, only a single reducing component showed its activity. Second, carbazole alkaloids are not soluble in aqueous medium. Therefore, we had to use SDS to prevent their precipitation in aqueous medium. Thus, in addition to $\mathbf{A}, \mathbf{B}, \mathbf{C}$, and $\mathbf{D}$, SDS might also play a role in the stabilization of these NPs. However, like AMEL, 


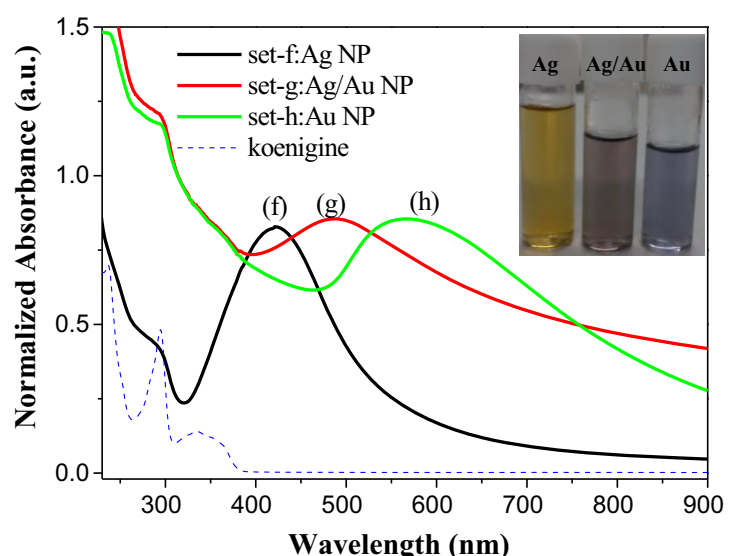

(i)

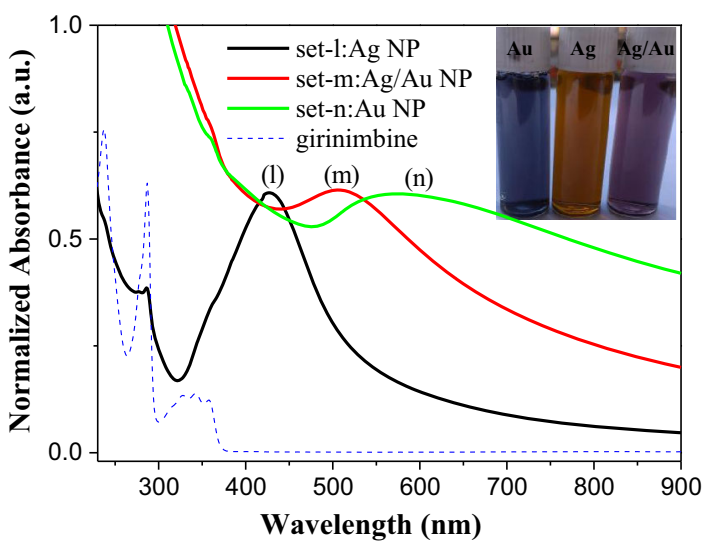

(iii)

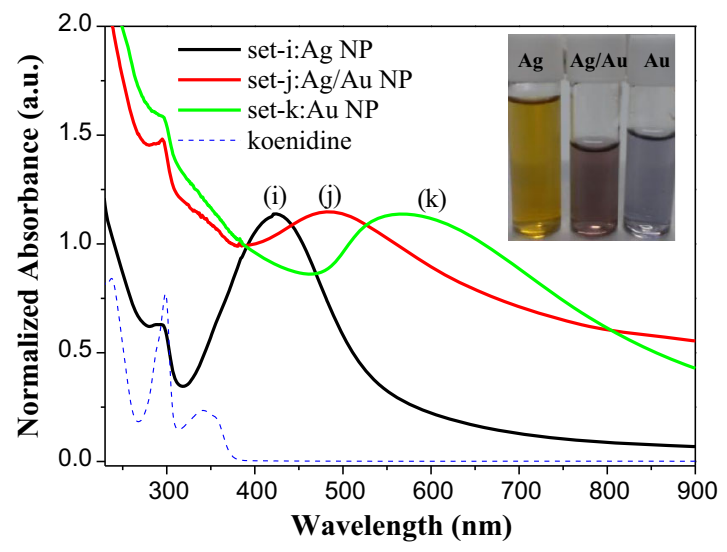

(ii)

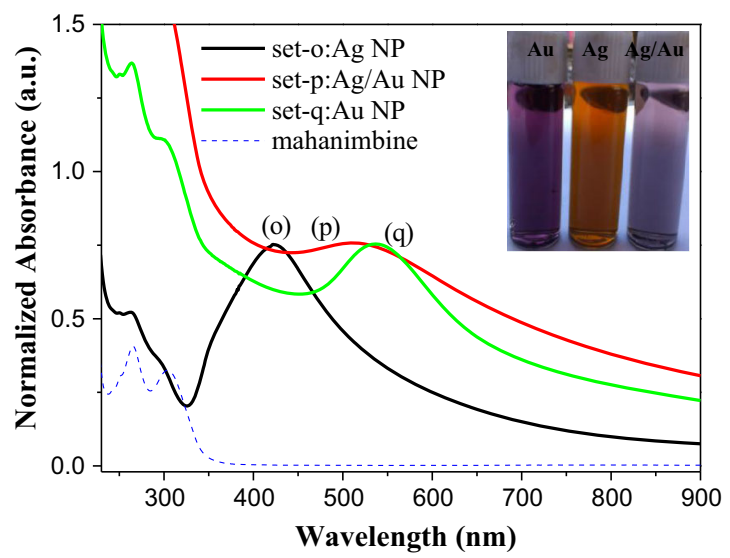

(iv)

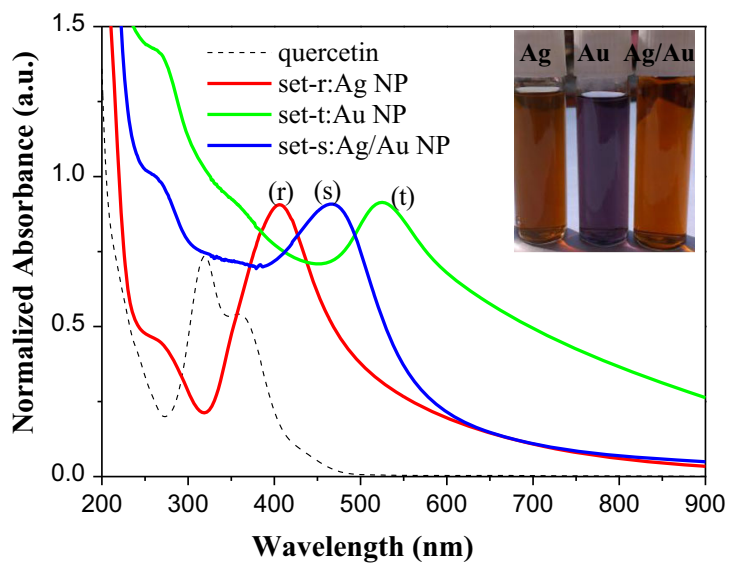

(v)

Fig. 3 i-v UV-Vis spectra and vi TEM image for the different sets of synthesized NPs: Ag NPs, Au NPs, and Ag/Au alloy (1:1) NPs prepared using koenigine (sets $f-h$ ), koenidine (sets $i-k$ ), girinimbine (sets $l-n$ ), mahanimbine (sets $o-q$ ), and quercetin (sets $r-t)$,

in these four cases, also, Ag NPs were found to be mostly uniform in size and spherical in shape [Fig. 3vi(sets $\mathrm{f}, \mathrm{i}, 1$, and o)], while anisotropic structures were quite visible in the case of Au NPs [Fig. 3vi(sets h, k, n, and $\mathrm{q})$ ]. respectively. Broken line in $\mathbf{i}-\mathbf{v}$ represents absorbance curve of koenigine, koenidine, girinimbine, mahanimbine, and quercetin, respectively. Inset in $\mathbf{i}-\mathbf{v}$ shows the color of the corresponding NP solutions

Interestingly, Ag and Au NPs synthesized by quercetin were found to be more uniform in size and NPs formed were smaller in size. In this case, Au NP showed a wide spectrum of morphology in addition to regular spherical shape [Fig. 3vi(sets $r$ and $t)$ ]. 
(set-f)
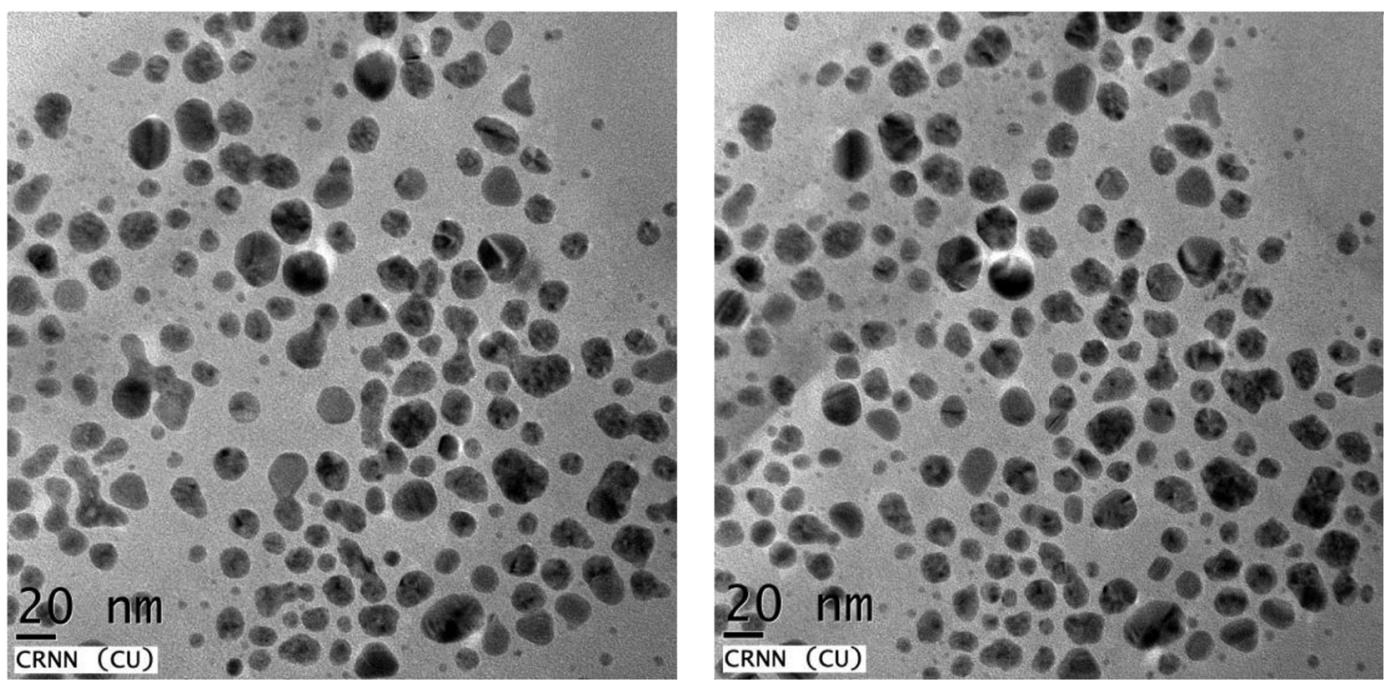

(set-g)
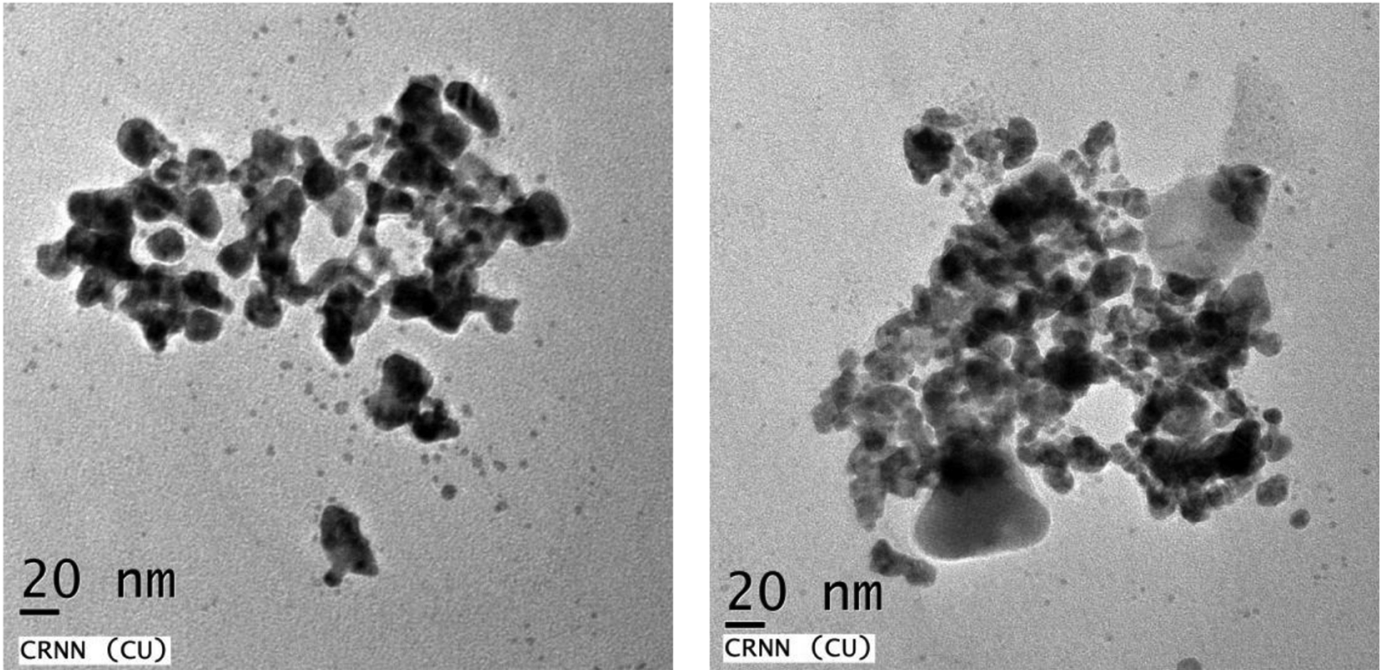

(set-h)
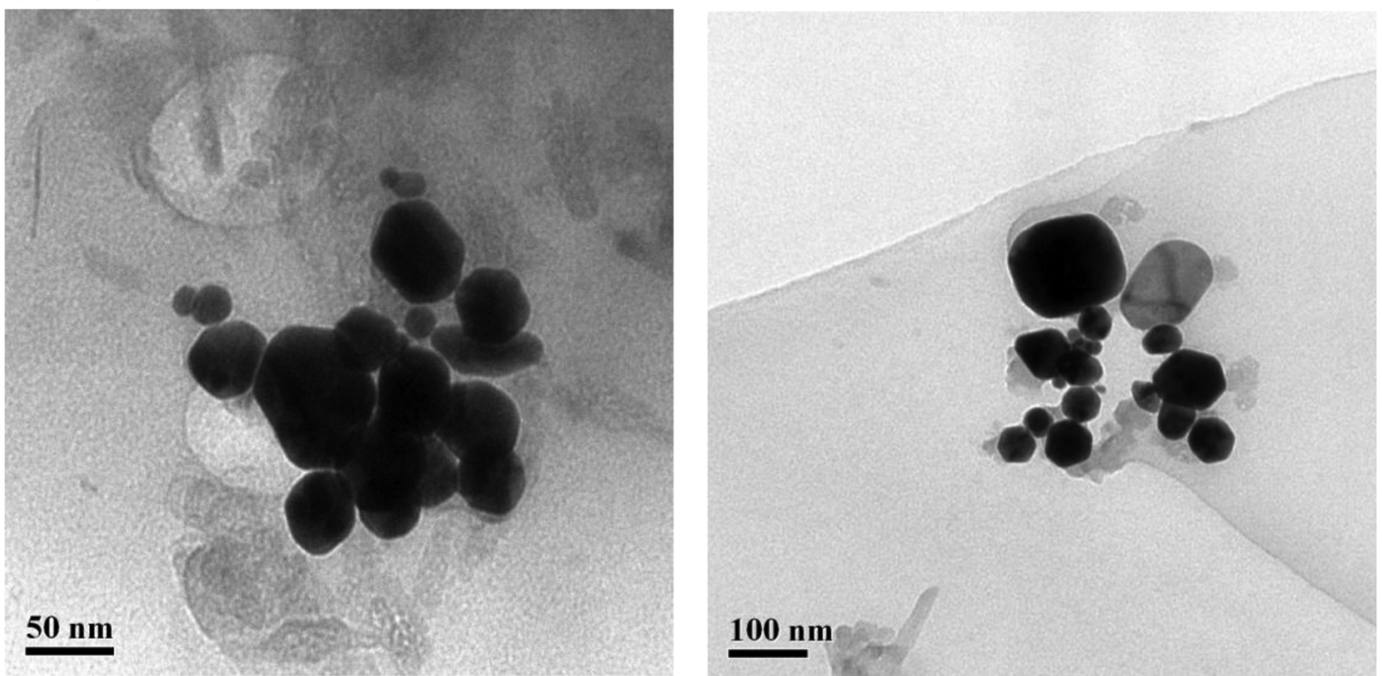

Fig. 3 continued 

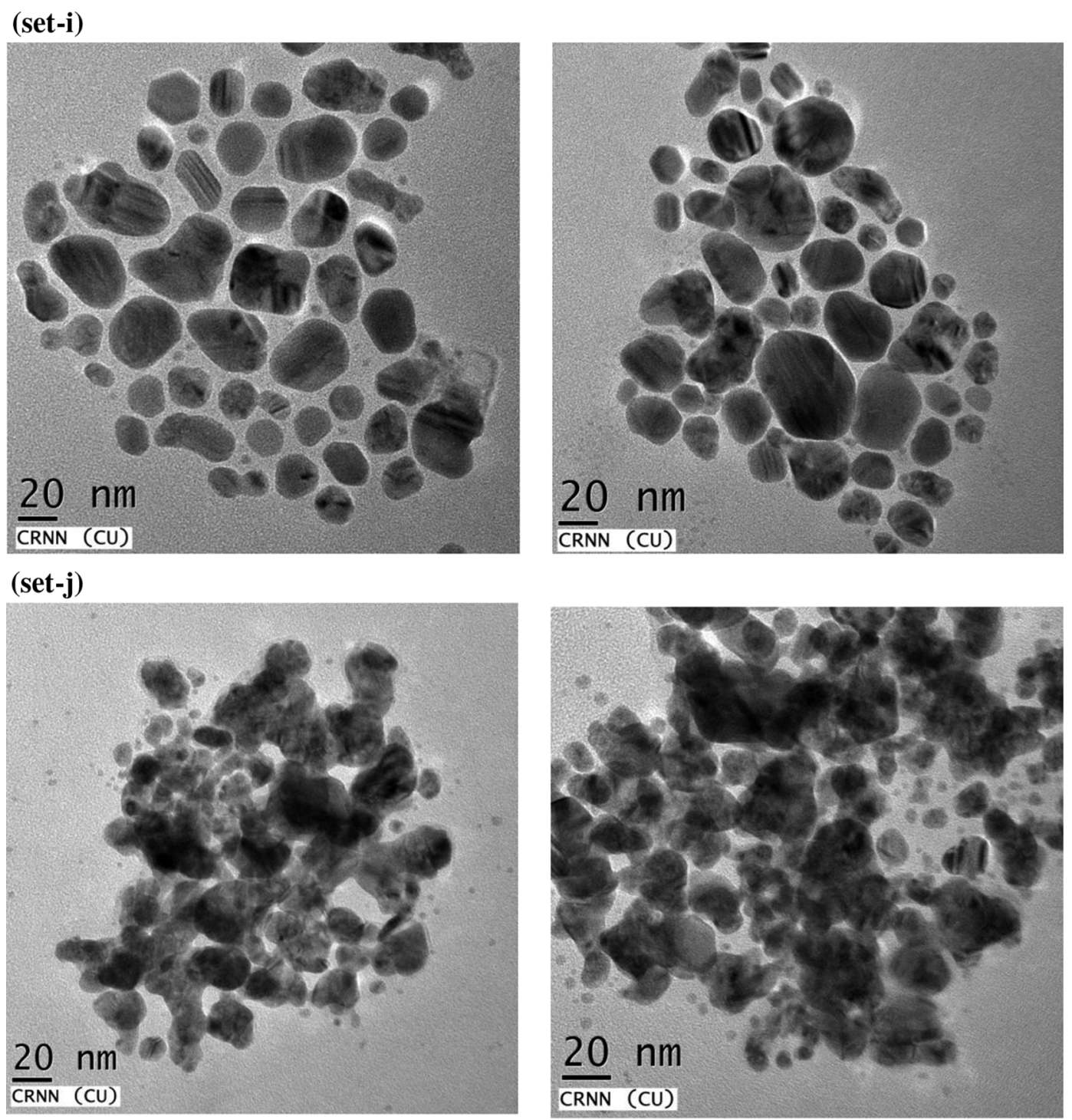

\section{(set-k)}
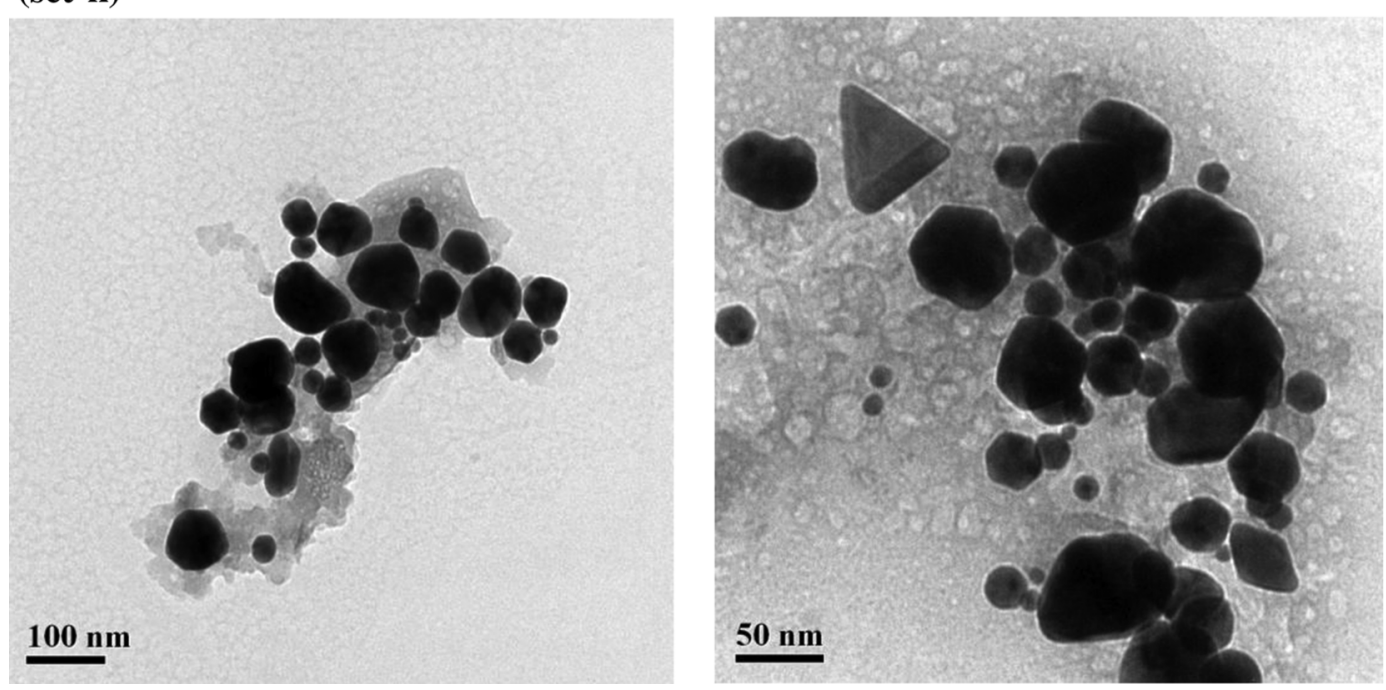

Fig. 3 continued 


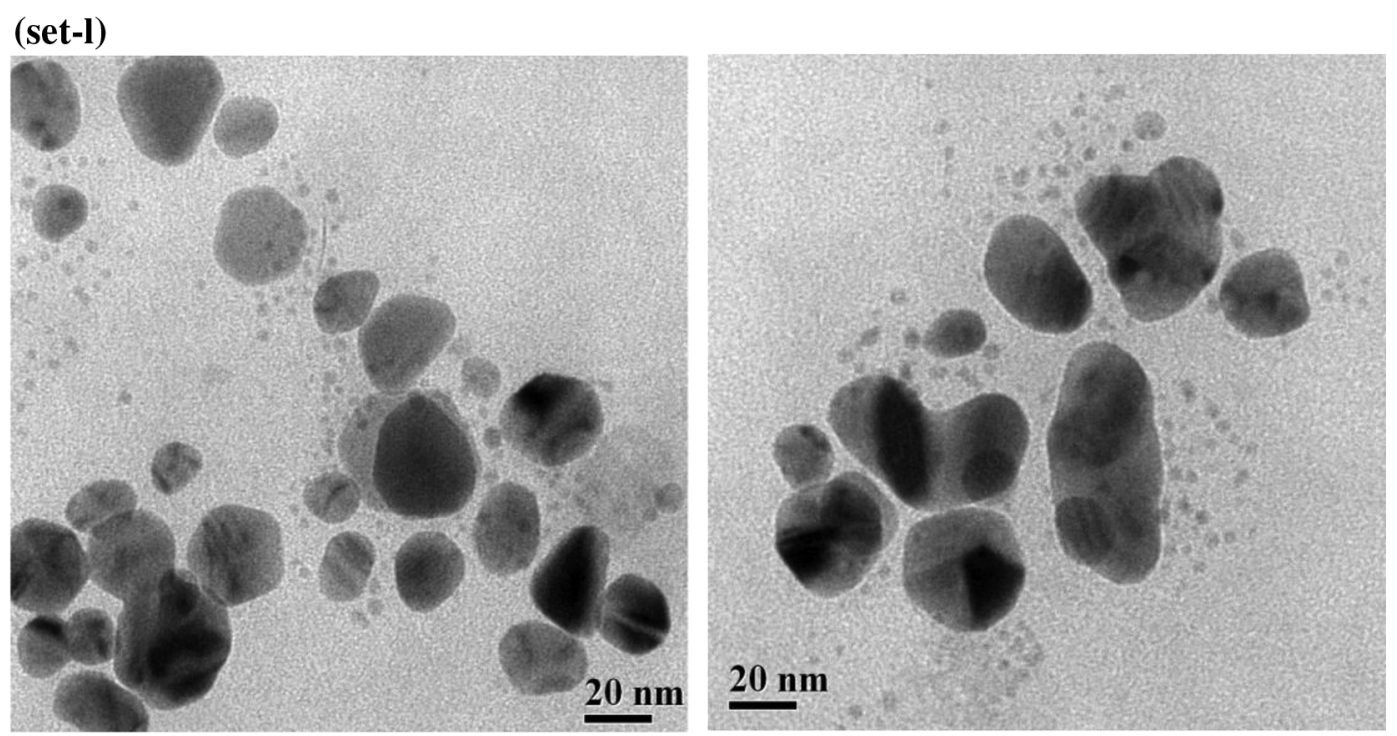

\section{(set-m)}
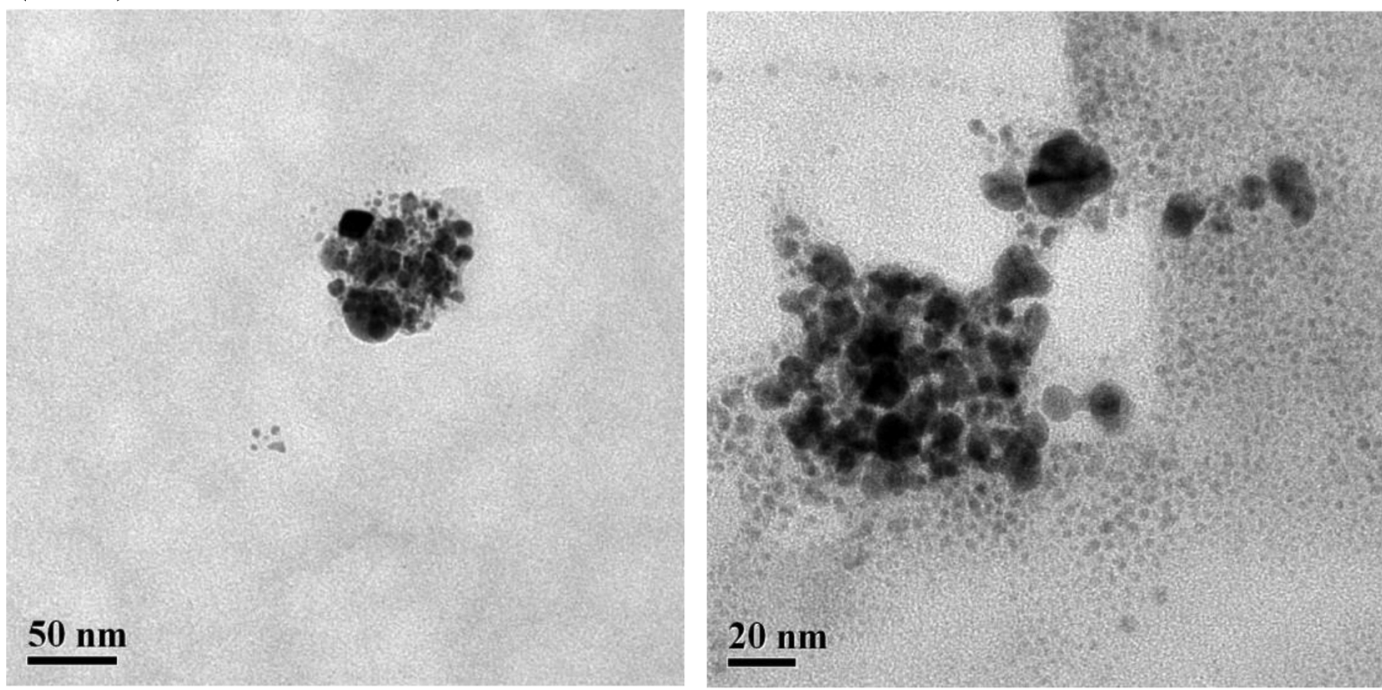

(set-n)
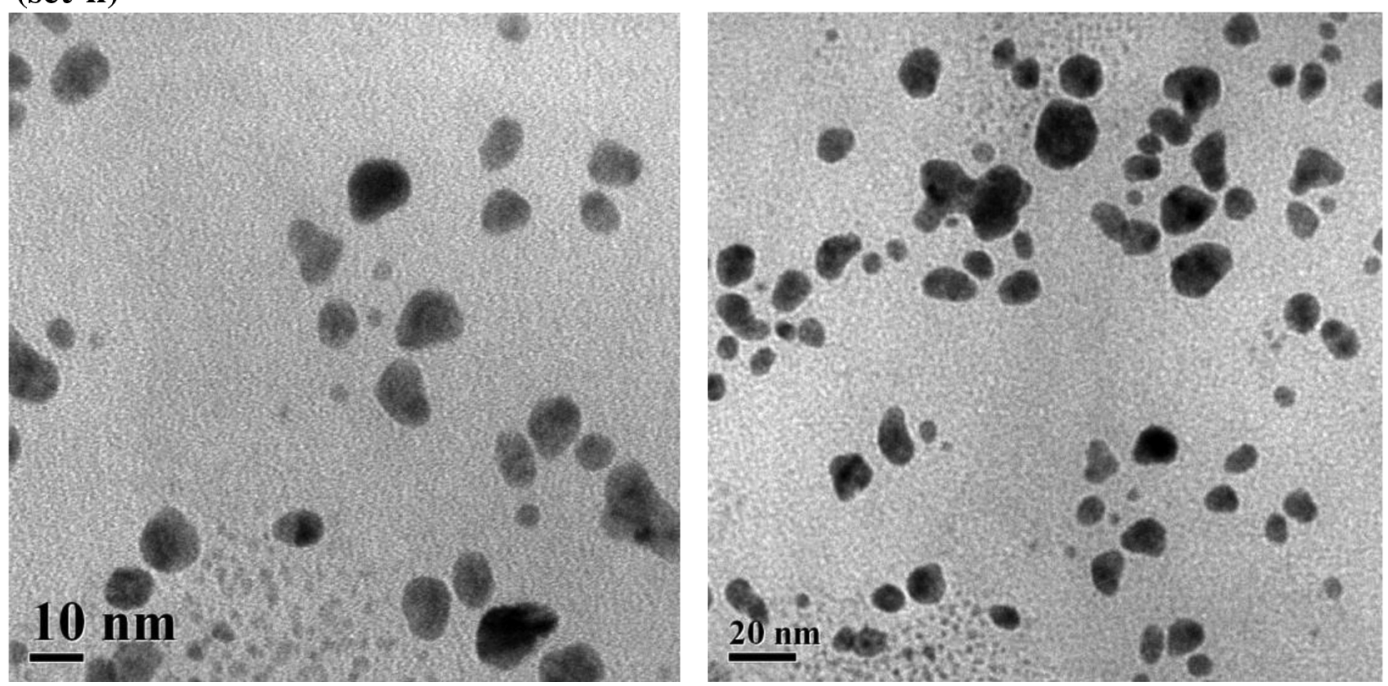

Fig. 3 continued 


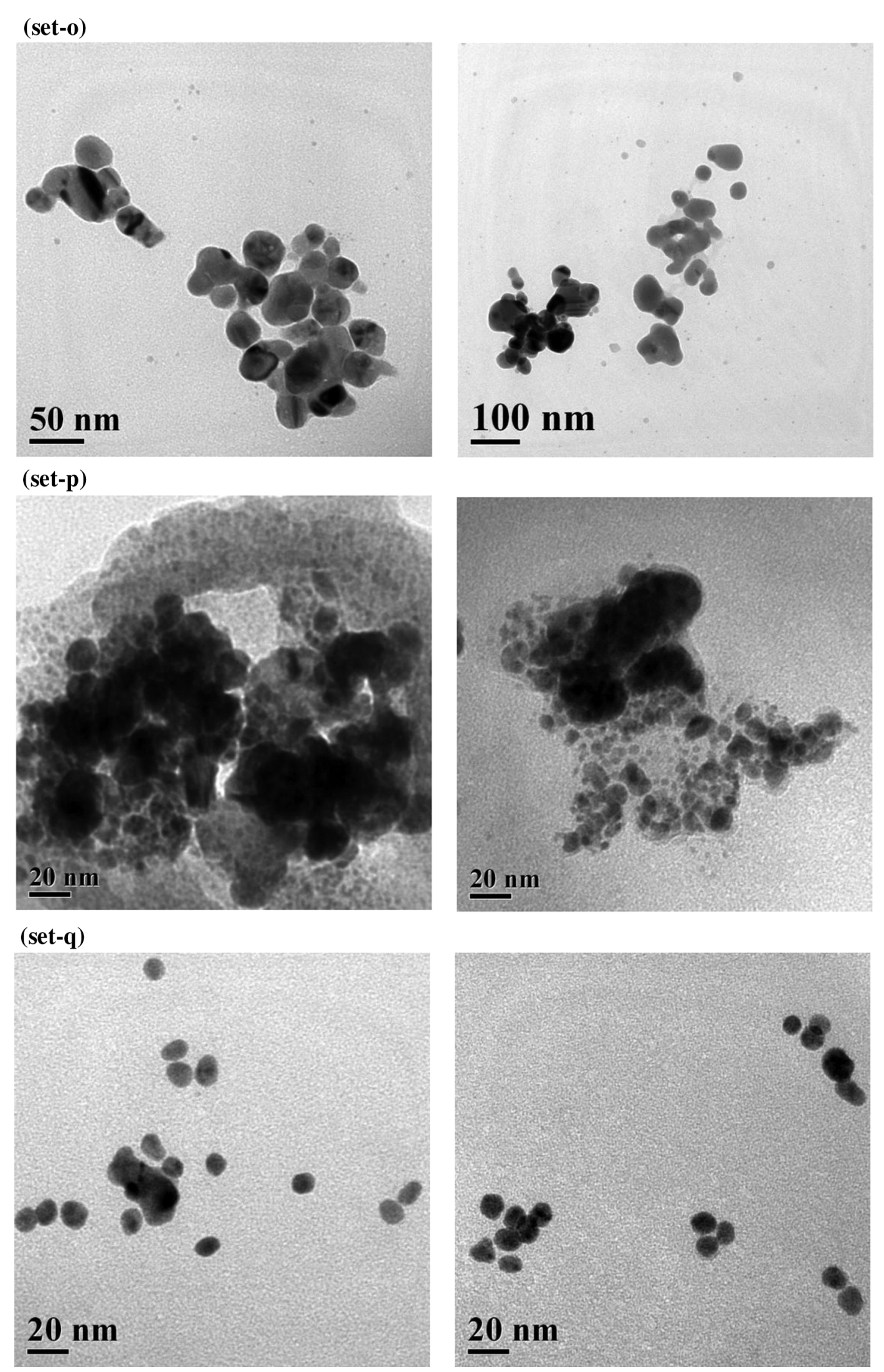

Fig. 3 continued 

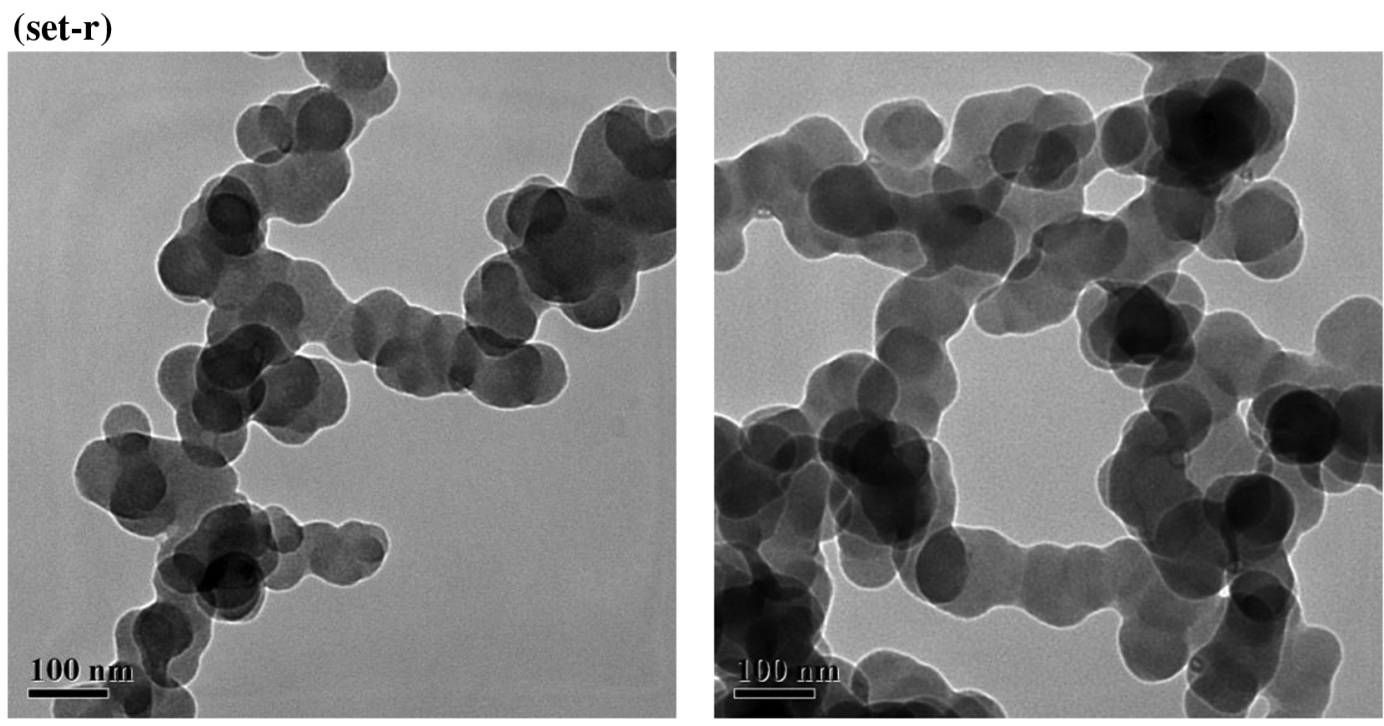

\section{(set-s)}
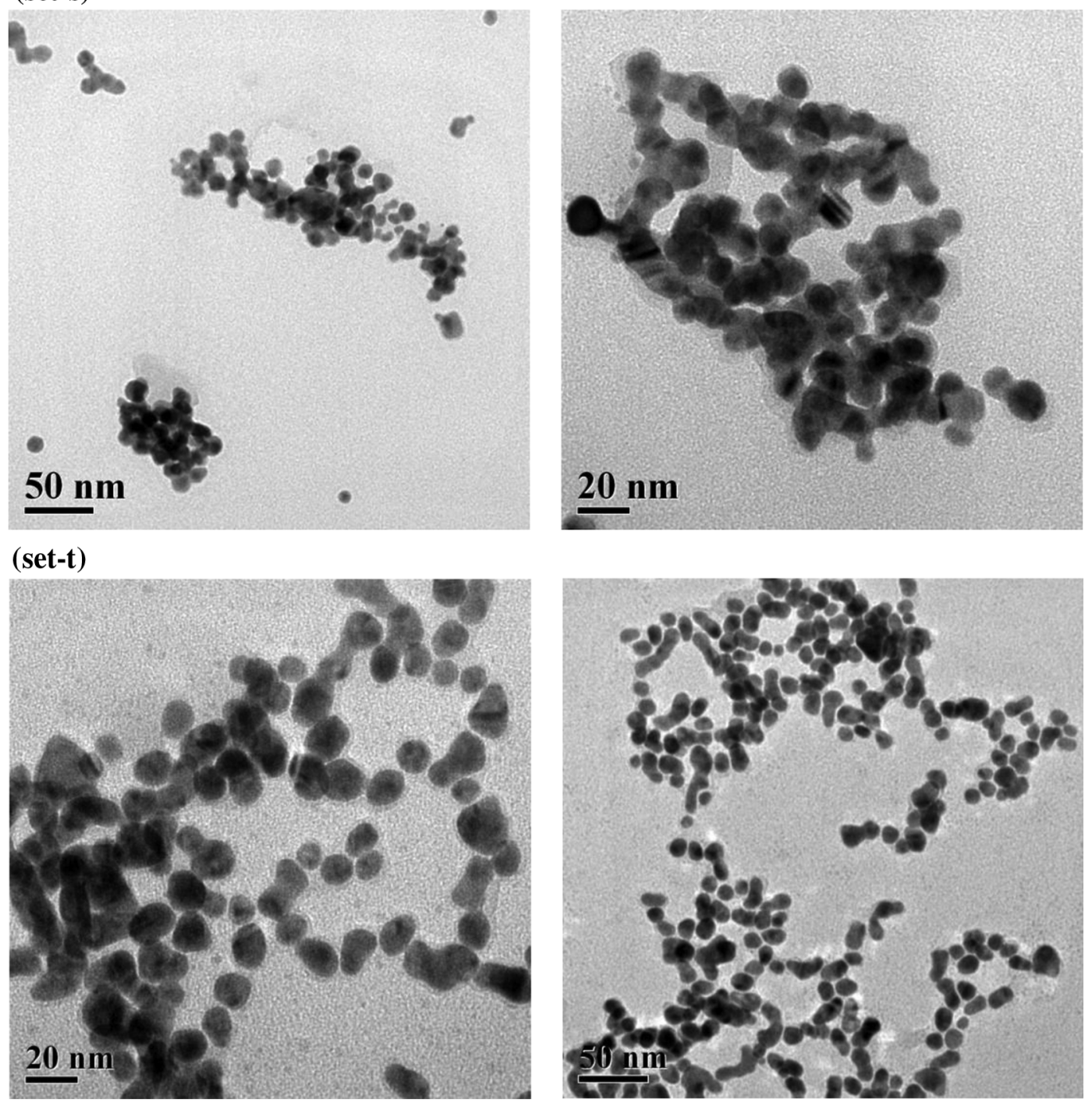

(vi)

Fig. 3 continued

Springer 
In all the cases, TEM images [Fig. 3vi(sets g, j, m, p, and s)] clearly confirmed the formation of $\mathrm{Ag} / \mathrm{Au}$ alloy NPs.

Selected area electron diffraction (SAED) patterns for $\mathrm{Ag}, \mathrm{Au}$, and $\mathrm{Ag} / \mathrm{Au}[(1: 1),(1: 3)$, and (3:1)] alloy NPs synthesized by the present method are shown in the insets of Fig. 2iv. The diffraction rings in SAED correspond to the crystalline nature of the synthesized NPs. The crystalline nature of the synthesized NPs was further confirmed from their powder X-ray diffraction (XRD) patterns, as shown in Fig. 2v. The presence of four lattice planes viz, (111), (200), (220), and (311) confirmed the formation of fcc Ag NP, and these lattice planes were associated with the diffraction peaks at almost $38.06^{\circ}, 43.78^{\circ}$, and $64.56^{\circ}$ [Fig. 2v(set a)]. On the other hand, the presence of fcc Au NPs in the sample was indicated by the appearance of four lattice planes (111), (200), (220), and (311) corresponding to the diffraction peak at $38.18^{\circ}, 44.39^{\circ}, 64.83^{\circ}$, and $77.61^{\circ}$ [Fig. 2v(set e)].

Four lattice planes (111), (200), (220), and (311) were associated with the diffraction peaks at $38.18^{\circ}, 43.92^{\circ}$, $64.71^{\circ}$, and $77.26^{\circ}$ for fcc $\mathrm{Ag} / \mathrm{Au}$ (1:1) alloy NP [Fig. 2$\mathrm{v}($ set $\mathrm{c})]$. These results confirmed the formation of $\mathrm{Ag}$ (metallic silver, JCPDS 04-0783), Au (metallic gold, JCPDS 04-0784), and for bimetallic Ag/Au (1:1) alloy NPs in pure phase.

Energy dispersive X-ray (EDX) studies of the synthesized NPs [Ag NP, Au NP, and Ag/Au NP (1:1)] were done to detect their elemental composition, as shown in Fig. 2vi. In all these EDX spectra, strong signals for copper $(\mathrm{Cu})$ were observed. This may be due to the presence of $\mathrm{Cu}$ in $\mathrm{Cu}$-grid used for the experiment.

\section{Understanding the roles of AMEL and its chemical constituents as GMA in controlling the surface structure of the synthesized monometallic (Ag and Au) and bimetallic (Ag/Au) NPs}

TGA analysis of the monometallic $\mathrm{Ag}, \mathrm{Au}$, and bimetallic $\mathrm{Ag} / \mathrm{Au}$ (1:1) alloy NPs synthesized by AMEL gave an idea about the thermal stability and surface adsorbed moieties of these NP sets. Results of TGA analysis are shown in Fig. 4i. The plot initially showed a decrease in weight up to $100{ }^{\circ} \mathrm{C}$ which may be due to the water molecules present with NPs. In all the cases, steady weight loss of NP samples was observed. Total $54 \%$ weight loss was observed for Ag NPs, while 34 and $36 \%$ weight losses were noticed in the case of $\mathrm{Au}$ and $\mathrm{Ag} / \mathrm{Au}$ (1:1) NPs, respectively [Fig. 4i(sets a,e, and c)]. Therefore, after elimination of water from the NP surface, this weight loss might have occurred due to the surface desorption of the active chemical constituents of AMEL which acted as the reducing agent and adhered on the surface of the NPs to give them stability and thus imparted a multifunctional activity to AMEL.

Elaborate IR spectroscopic measurements of each of the systems involved in the present protocol gave an idea about the surface chemistry of the synthesized NPs. In the case of AMEL itself (before the reduction of either $\mathrm{Ag}^{+}$to $\mathrm{Ag}^{0}$ or $\mathrm{Au}^{3+}$ to $\mathrm{Au}^{0}$ ), IR peaks observed were: $1711,1613,1393,1216,1142$, and $1060 \mathrm{~cm}^{-1}$. The IR peaks at 1711 and $1613 \mathrm{~cm}^{-1}$ may be associated with the stretching vibrations for $-\mathrm{C}=\mathrm{O}$ (keto and ester) groups and for bending mode of vibration of $\mathrm{N}-\mathrm{H}$ (amine) groups. On the other hand, IR peaks at 1393, 1216, 1142, and $1060 \mathrm{~cm}^{-1}$ can be associated with stretching vibrations for $-\mathrm{C}=\mathrm{C}-[$ (in-ring) aromatic $], \mathrm{C}-\mathrm{O}$ (ester, ether), $\mathrm{C}-\mathrm{O}$ (polyol), and $\mathrm{C}-\mathrm{N}$ (amine), respectively [Fig. 4ii (for AMEL, sets a, e, and c)]. These result further confirmed that the extract (AMEL) was rich in oxygen and nitrogen containing secondary metabolites which can be polyphenols, flavonoids (e.g., quercetin), and alkaloids, such as carbazole alkaloid [e.g., koenigine (A), koenidine $(\mathbf{B})$, girinimbine $(\mathbf{C})$, and mahanimbine (D) which we have also isolated from AMEL].

As a whole, IR spectra of $\mathrm{Ag}, \mathrm{Au}$, and $\mathrm{Ag} / \mathrm{Au}$ (1:1) alloy NPs moderately resembled the IR spectrum of AMEL itself. However, some new peaks appeared with slight shifting in the case of AMEL itself. These were found to be at: $1709,1600,1385$, and $1039 \mathrm{~cm}^{-1}$ (for Au NPs); 1729, $1614,1450,1383$, and $1073 \mathrm{~cm}^{-1}$ (for Ag NPs), and 1726, $1623,1444,1342,1210$, and $1078 \mathrm{~cm}^{-1}$ (for $\mathrm{Ag} / \mathrm{Au}(1: 1)$ alloy NPs).

In the present case, we have not used any external reducing and stabilizing agent. Therefore, it is clear that AMEL itself played both these two roles and the chemical components of AMEL imparted these characteristics to AMEL. These chemical components not only reduced $\mathrm{Ag}^{+}$ to $\mathrm{Ag}^{0}$ or $\mathrm{Au}^{3+}$ to $\mathrm{Au}^{0}$, but at the same time stabilized corresponding NPs either in their free form or in other oxidized form. Close resemblance of the FT-IR spectrum of the AMEL itself with NPs systems synthesized by it may be due to the fact that the unreacted chemical components of AMEL along with their reacted forms adhered on the surface of the NPs giving them stability. For further understanding of these facts, we have also done detailed IR spectroscopic studies of the individual chemical components [koenigine (A), koenidine (B), girinimbine (C), mahanimbine (D), and quercetin] and the NPs synthesized by these components of AMEL. The results are shown in Figs. S1-S6 of the Electronic Supplementary Material (pages S11-13). These results further confirmed the fact that the component actively took part in the reduction process as well as in the surface functionalization of these NPs. 


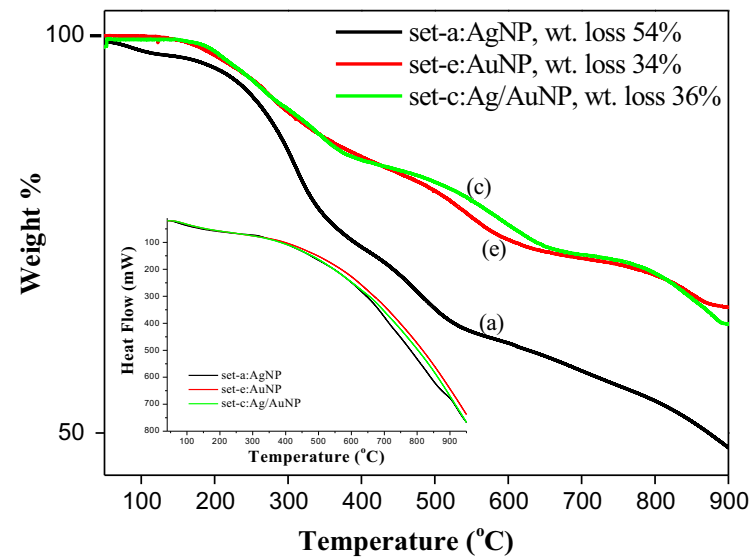

(i)

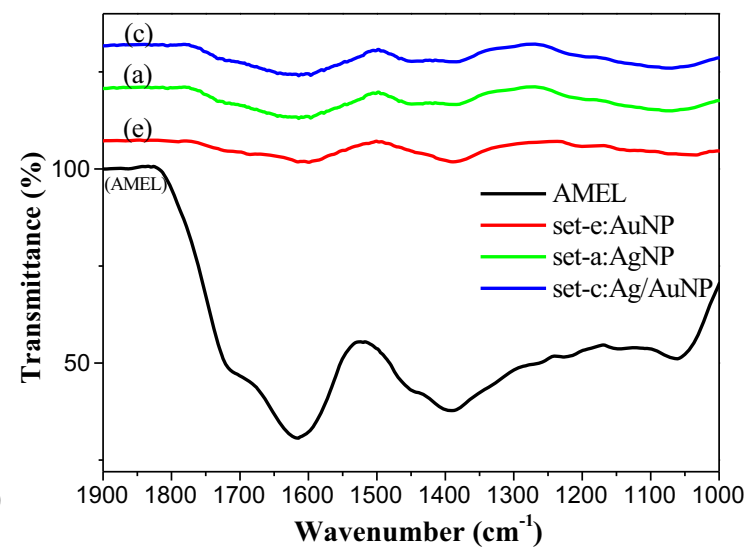

(ii)

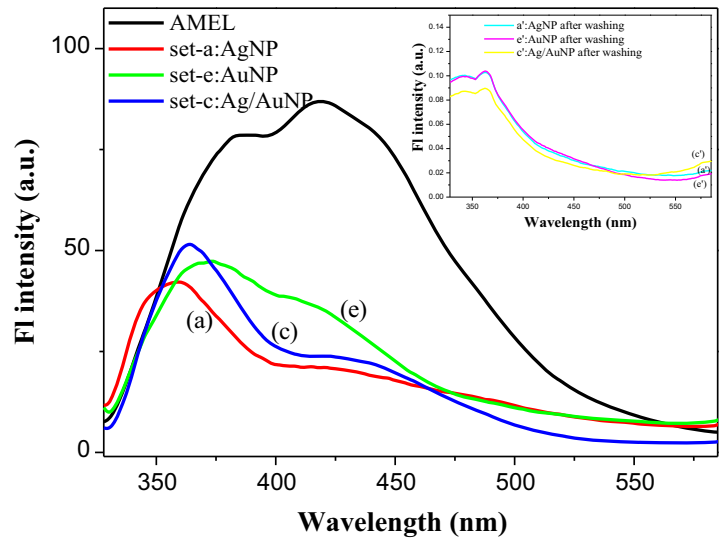

(iii)

Fig. 4 i TGA plot for different sets of NPs synthesized by AMEL as GMA: Ag NP (set a), Ag/Au alloy NP (1:1) (set c), and Au NP (set e). Inset of $\mathbf{i}$ shows the corresponding DTA plots. ii FT-IR spectra of different sets of NPs and AMEL itself. iii Fluorescence spectra of AMEL before and after the formation of Ag NPs (set a), Ag/Au alloy

Role of AMEL and its chemical components on the surface functionalization of the synthesized NPs were further elaborated with the help of fluorescence spectroscopic measurements. The fluorescence spectra of $\mathrm{Ag}$ $\mathrm{NP}, \mathrm{Au} \mathrm{NP}$, and $\mathrm{Ag} / \mathrm{Au}$ (1:1) NP solution synthesized by AMEL in aqueous medium are shown in Fig. 4iii(sets a, $\mathrm{e}$, and $\mathrm{c})$. The fluorescence emission maximum $\left[\lambda_{\mathrm{em}}\right.$ (max)] for Ag NP was observed at $350 \mathrm{~nm}$, whereas $\lambda_{\mathrm{em}}$ (max) of $\mathrm{Au} \mathrm{NP}$ was observed at $430 \mathrm{~nm}$. However, in the case of $\mathrm{Ag} / \mathrm{Au}(1: 1)$ alloy $\mathrm{NP}, \lambda_{\mathrm{em}}(\max )$ was noticed at $364 \mathrm{~nm}$ with a broad hump at $430 \mathrm{~nm}$ [Fig. 4iii(sets a, $\mathrm{e}$, and $\mathrm{c})]$.

Similar type of fluorescence behavior was observed for the NP systems synthesized by the individual chemical components, as shown in Fig. S7 of the Electronic Supplementary Material (page S14).
NPs (1:1) (set c) and Au NPs (set e). Inset of iii shows the fluorescence spectra of NPs after repeated washing: $\operatorname{Ag~NP}\left(a^{\prime}\right), \mathrm{Ag} /$ Au alloy NP (1:1) $\left(c^{\prime}\right)$, and Au NP $\left(e^{\prime}\right)$. Excitation wavelength was fixed at $308 \mathrm{~nm}$

In the case of aqueous solution of AMEL itself, the appearance of broad emission band [Fig. 4iii (AMEL)] indicated the presence of several fluorophoric moieties in it and their combined effect was reflected in the broad nature of the fluorescence emission band of this system. In the case of single fluorophoric system, such as koenigine (A), koenidine (B), girinimbine (C), and mahanimbine (D) along with quercetin and the NP sets synthesized by them, the respective emission band maxima were found to be comparatively sharper [Figs. S7 and S8 of the Electronic Supplementary Material (page S14)]. After repeated washing of these NPs, the fluorescence activity was found to be diminished [inset of Fig. 4iii and Fig. S9 of the Electronic Supplementary Material, page S15].

Active component, such as carbazole alkaloids and polyphenols, flavonoids of AMEL have strong fluorophoric 

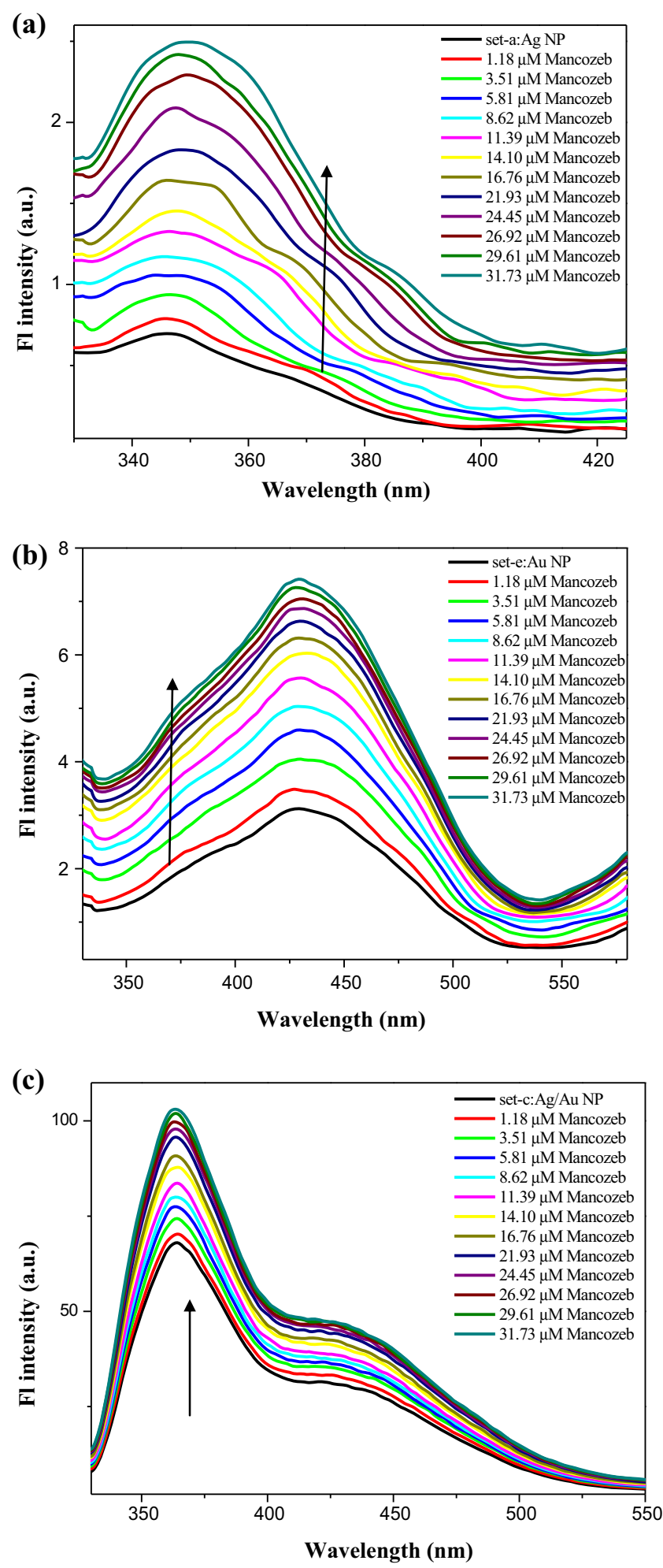

(i)

Fig. 5 Mancozeb sensing activity of monometallic Ag, $\mathrm{Au}$ and bimetallic $\mathrm{Ag} / \mathrm{Au}$ (1:1) NPs synthesized by AMEL. Mancozeb sensing activity of different sets of NPs synthesized by i AMEL ( $a-$ $c$ ), ii koenidine $(a-c)$, and iii quercetin $(a-c)$. For studying this
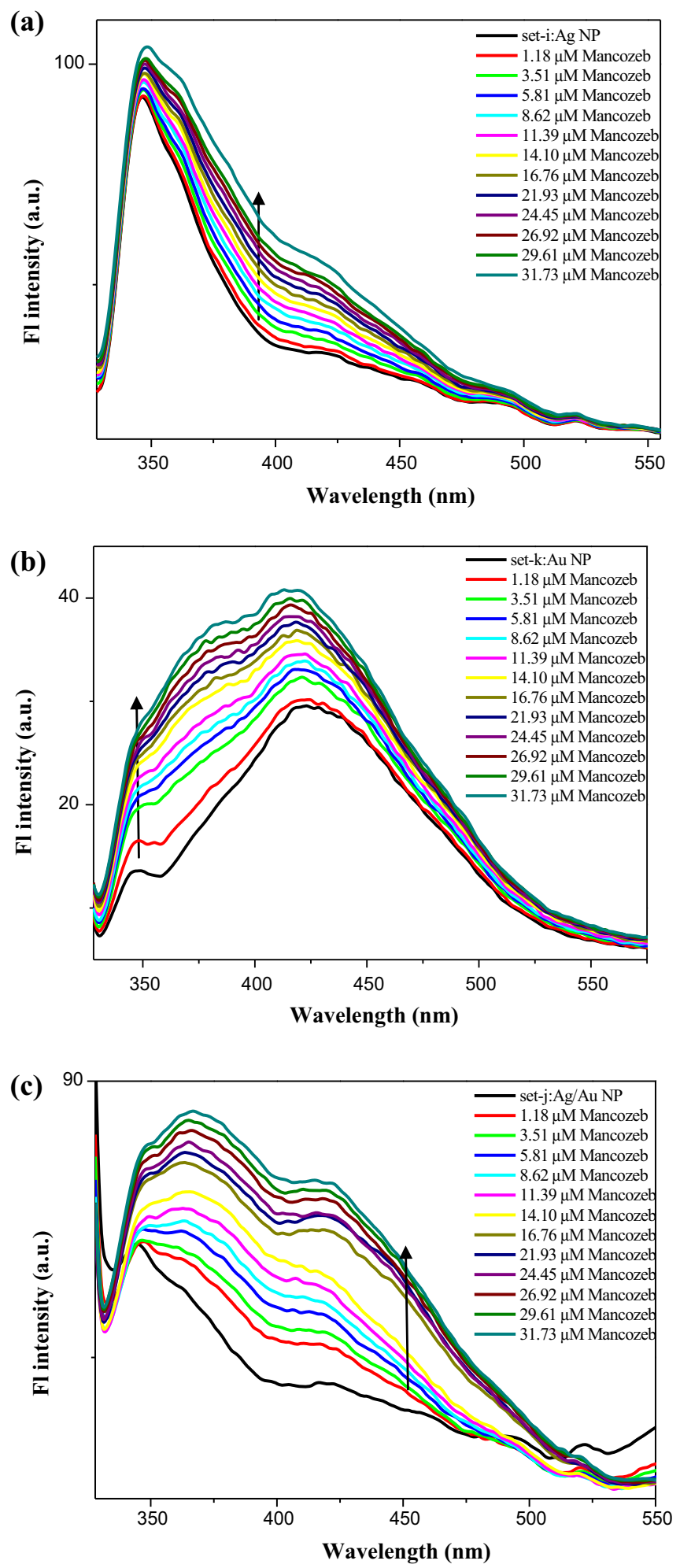

(ii)

sensing activity, fluorescence spectra of different sets of synthesized NPs were recorded in the absence and presence of different concentrations $(1.18-31.73 \mu \mathrm{M})$ of aqueous solution of mancozeb. Excitation wavelength was fixed at $308 \mathrm{~nm}$ 

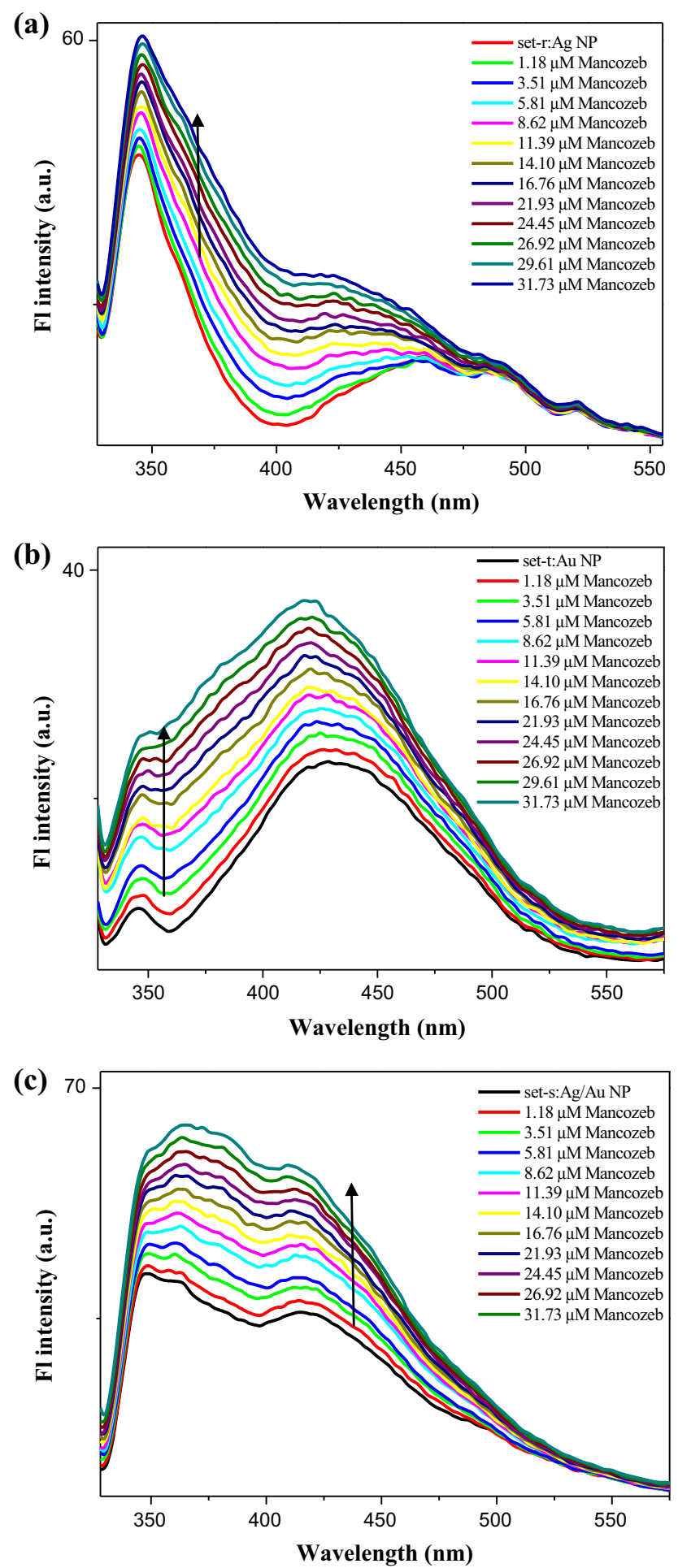

(iii)

Fig. 5 continued

property [31] and these molecules imposed characteristics fluorescence activity to the synthesized NP systems and leaded in controlling the surface structure of the synthesized NPs.
Exploring the efficacy of the monometallic (Ag and Au) and bimetallic (Ag/Au) NPs synthesized by AMEL and its chemical constituents as GMA towards the fluorometric sensing of Mancozeb

We have tested the fluorescence sensing activity of the $\mathrm{Ag}, \mathrm{Au}$, and $\mathrm{Ag} / \mathrm{Au}$ (1:1) alloy NPs synthesized by AMEL towards the sensing of Mancozeb in aqueous medium. During the addition of Mancozeb to NP solution, a significant enhancement of the fluorescence intensity of each of the NP sets was observed (Fig. 5i-iii). The fluorescence emission maximum $\left[\lambda_{\mathrm{em}}(\max )\right]$ for Ag NP (set a), Au NP (set e), and Ag/Au (1:1) alloy NP (set c) systems (synthesized by AMEL) on the addition of Mancozeb solution were observed at 350,430 , and $364 \mathrm{~nm}$ with a broad hump at $430 \mathrm{~nm}$, respectively [Fig. 5i(a-c)].

We have also tested the fluorometric sensing activity of Ag NP, Au NP, and Ag/Au (1:1) alloy NPs synthesized by koenidine (B) and quercetin towards Mancozeb in aqueous medium, as shown in Fig. 5ii and iii, respectively. In these cases also, rapid enhancement of fluorescence intensity was observed on the addition of Mancozeb solution. These two compounds represent two different classes of natural products (carbazole alkaloid and polyhydroxy flavonoid, respectively) present in AMEL. The fluorescence behavior of AMEL was found to be the combination of the fluorescence characteristics of both these two classes [Fig. 4iii (AMEL) and Fig. S8 (page S14)].

During the present study, we have found that neither AMEL itself nor its any chemical constituents selected for the present work showed fluorometric/colorimetric sensing activity towards Mancozeb [Fig. S10 of the Electronic Supplementary Material, (page S15)]. We have also tested the sensing activity of AMEL and its chemical constituents in the presence of $\mathrm{Ag}, \mathrm{Au}$, and $\mathrm{Ag} / \mathrm{Au}$ (1:1) alloy NPs synthesized by chemical reduction method. However, in this case, negative results were obtained.

Therefore, it is very much clear that surface functionalization of these NP systems by AMEL or its chemical constituents was very much important for showing their fluorescence sensing activity towards Mancozeb. This enhancement of fluorescence emission intensity of the NP solution may be due to the fluorescence 'turn on' phenomenon [32]. Mancozeb itself was non-fluorescent. On gradual addition of Mancozeb to NP solution, the S-containing functional group of Mancozeb interacted more strongly with the NP surface by soft-soft interaction mode which helped Mancozeb moieties to remain anchored on the surface of the NPs more strongly than the fluorescent chemical components of AMEL and these strong fluorophoric moieties became free from the surface of NPs. Ultimately, this phenomenon increased the concentration of the fluorophoric moieties in the solution which rapidly enhanced or 'turned on' the fluorescence activity of the whole system (Fig. 5). 

representation of fluorescence sensing study of GMA synthesized nanoparticles (NPs) towards Mancozeb, a dithiocarbamate pesticide
Scheme 1 Schematic

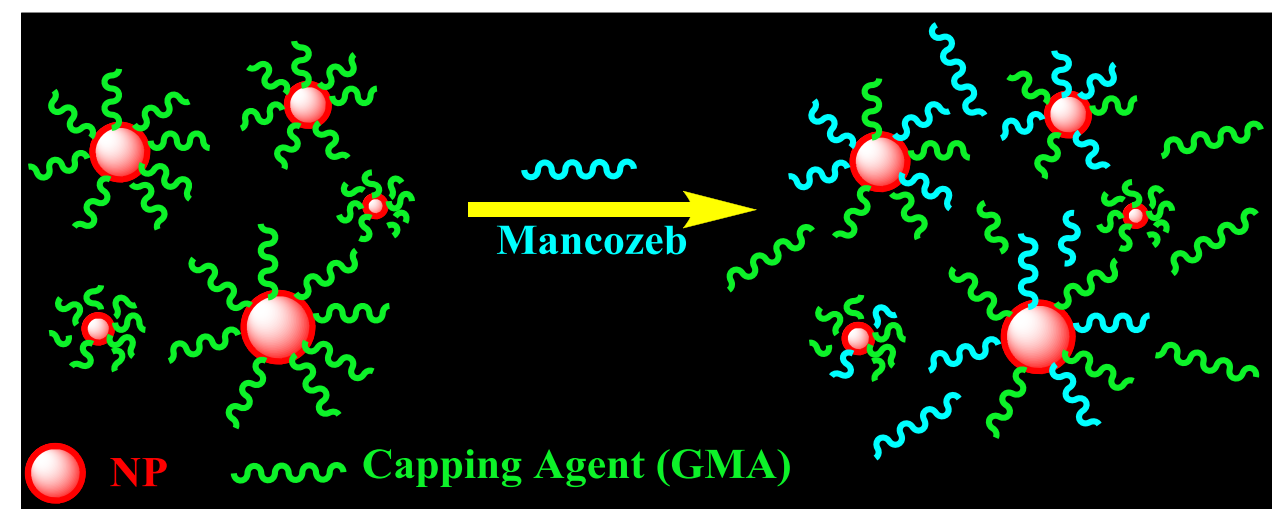

Table 3 Zeta-potential data of the synthesized NP and corresponding NP-Mancozeb systems

\begin{tabular}{lllll}
\hline Reducing agents & Set of NP & NP composition & Zeta potentials $(\mathrm{mV})$ \\
\cline { 3 - 5 } & & & In absence of mancozeb & In presence of mancozeb \\
\hline AMEL & $\mathrm{a}$ & $\mathrm{Ag} \mathrm{NP}$ & -31.0 & -21.3 \\
& $\mathrm{c}$ & $\mathrm{Ag} / \mathrm{Au} \mathrm{NP}$ & -41.6 & -17.4 \\
\multirow{3}{*}{ Koenidine } & $\mathrm{e}$ & $\mathrm{Au} \mathrm{NP}$ & -28.3 & -19.4 \\
& $\mathrm{i}$ & $\mathrm{Ag} \mathrm{NP}$ & -24.2 & -15.4 \\
& $\mathrm{j}$ & $\mathrm{Ag} / \mathrm{Au} \mathrm{NP}$ & -17.9 & -15.5 \\
Quercetin & $\mathrm{k}$ & $\mathrm{Au} \mathrm{NP}$ & -31.1 & -13.7 \\
& $\mathrm{r}$ & $\mathrm{Ag} \mathrm{NP}$ & -21.2 & -16.9 \\
& $\mathrm{~S}$ & $\mathrm{Ag} / \mathrm{Au} \mathrm{NP}$ & -22.2 & -19.3 \\
& $\mathrm{t}$ & $\mathrm{Au} \mathrm{NP}$ & -18.2 & -13.7 \\
\hline
\end{tabular}

Zeta potential of AMEL, koenidine, quercetin, and mancozeb is $-19.5,-16.4,-21.3$, and $-6.52 \mathrm{mV}$, respectively
The sensing mechanism is shown in Scheme 1.

The attachment of Mancozeb on the NPs surface was also confirmed from the zeta-potential values of these NP solutions (Table 3). It was observed that after the addition of Mancozeb to the NP solutions, the increase (more positive) in zeta-potential value was observed. This is due to the attachment of Mancozeb molecules, which have more positive zeta-potential value $(-6.52 \mathrm{mV})$ to the NP surface by replacing AMEL or its chemical compounds, such as koenidine or quercetin which have more negative zeta-potential $(-19.5,-16.4$, and $-21.3 \mathrm{mV}$, respectively) (Table 3 ).

\section{Conclusions}

In conclusion, we have used an indigenous source (as leaves of Indian curry leaf plant, a well-known Indian medicinal plant along with its chemical components) as GMA to develop energy-efficient, economically viable and environment-friendly synthetic protocols for stable, crystalline, and fluorescent monometallic ( $\mathrm{Ag}$ and $\mathrm{Au}$ ) and also bimetallic ( $\mathrm{Ag} / \mathrm{Au}$ alloy) nanoparticles with wide spectrum of controlled and target specific morphologies under sonochemical condition. These NPs showed their extreme efficacy towards the fluorometric sensing of a dithiocarbamate pesticide, Mancozeb in aqueous medium. AMEL (aqueous methanol extract of leaves) itself along with its active chemical constituents having characteristic fluorescent activity controlled the surface chemistry of the synthesized NPs and also imposed specific fluorescent behavior (in situ) to them. This opened up their possible utility as the eco-friendly and easily synthesizable fluorometric sensors and detectors for the hazardous dithiocarbamate pesticides/fungicide, such as Mancozeb (extensively used in agriculture industries) in aqueous medium. Moreover, the use of non-toxic (leaves are edible) GMA may render these NPs biocompatibility, and thus, these NPs can be explored in the future for the in vivo detection of toxic fluorescent compounds.

Acknowledgements We thank SERB-DST [sanction order no. SR/ SO/BB-0007/2011 dated 21.08.2012 to N. A. B.] for the financial support. M. N. A., S. B., and S. D. thanks SERB-DST, MANF-UGC, and CSIR, respectively, for their fellowships. We thank the Department of Chemistry, Siksha Bhavana, Visva-Bharati (Central University) and its DST-FIST and UGC-SAP (Phase-II) programmes for necessary infrastructural and instrumental facilities. We also thank the 
Department of Physics, Siksha Bhavana, Visva-Bharati (Central University) for powder XRD analysis. We acknowledge Professor T. Basu in the Department of Bio-chemistry and Bio-physics, University of Kalyani, Kalyani-741235, W.B., India for his kind help in zetapotential measurements. The acknowledgement is also due to the CRNN, University of Calcutta and IIT Kharagpur, W.B., India for the TEM facility.

\section{Compliance with ethical standards}

Conflict of interest The authors declare no competing financial interest.

Open Access This article is distributed under the terms of the Creative Commons Attribution 4.0 International License (http://creativecommons. org/licenses/by/4.0/), which permits unrestricted use, distribution, and reproduction in any medium, provided you give appropriate credit to the original author(s) and the source, provide a link to the Creative Commons license, and indicate if changes were made.

\section{References}

1. Rao, C.N.R., Kulkarni, G.U., Thomas, P.J., Edwards, P.P.: Sizedependent chemistry: properties of nanocrystals. Chem. Eur. J. 8, 28-35 (2002)

2. Sanchez-Ramirez, J.F., Pal, U., Nolasco-Hernandez, L., Mendoza-A' lvarez, J., Pescador-Rojas, J.A.: Synthesis and optical properties of $\mathrm{Au}-\mathrm{Ag}$ nanoclusters with controlled composition. J. Nanomater. 2008, 1-9 (2008)

3. Mondal, S., Roy, N., Laskar, R.A., Basu, S., Mandal, D., Begum, N.A.: Biogenic synthesis of $\mathrm{Au}, \mathrm{Ag}$ and bimetallic $\mathrm{Au} / \mathrm{Ag}$ nanopaticles using mahogany (Swietenia mahogany JACQ.) leaf extract. Colloids Surf. B Biointerf. 82, 497-504 (2011)

4. Roy, N., Mondal, S., Laskar, R.A., Basu, S., Mandal, D., Begum, N.A.: Biogenic synthesis of $\mathrm{Au}$ and $\mathrm{Ag}$ nanoparticles by Indian propolis and its constituents. Colloids Surf. B Biointerf. 76, 317-325 (2010)

5. Shiv Shankar, S., Rai, A., Ahmad, A., Sastry, M.: Rapid synthesis of $\mathrm{A}, \mathrm{Ag}$ and bimetallic Au core-Ag shell nanoparticles using neem (Azdirachta indica) leaf broth. J. Colloid Interf. Sci. 275, 496-502 (2004)

6. Alam, MdN, Chatterjee, A., Das, S., Batuta, S., Mandal, D., Begum, N.A.: Burmese grape fruit juice can trigger the "logic gate" like colorimetric sensing behavior of $\mathrm{Ag}$ nanoparticles towards toxic metal ions. RSC Adv. 5, 23419-23430 (2015)

7. Thakkar, K.N., Mhatre, S.S., Parikh, R.Y.: Biological synthesis of metallic nanoparticles. Nanomedicine. 6, 257-262 (2010)

8. Nadagouda, M.N., Varma, R.S.: Green and controlled synthesis of gold and platinum nanomaterials using vitamin $\mathrm{B}_{2}$ : densityassisted self-assembly of nanospheres, wares and rods. Green Chem. 8, 516-518 (2006)

9. Anastas, P.T., Warner, J.C.: Green chemistry: theory and practice, p. 30. Oxford University Press, New York (1998)

10. Raveendran, P., Fu, J., Wallen, S.L.: Completely "Green" synthesis and stabilization of metal nanoparticles. J. Am. Chem. Soc. 125, 13940-13941 (2003)

11. Shiv Shankar, S., Rai, A., Ahmad, A., Sastry, M.: Controlled the optical properties of lemongrass extract synthesized gold nanotriangles and potential application in infrared-absorbing optical coatings. Chem. Mater. 17, 566-572 (2005)

12. Chandran, S.P., Chaudhary, M., Pasricha, R., Ahmad, A., Sastry, M.: Synthesis of gold triangles and silver nanoparticles using Aloe vera plant extract. Biotechnol. Prog. 22, 577-583 (2006)
13. Huang, J., Li, Q., Sun, D., Lu, Y., Su, Y., Yang, X., Wang, H., Wang, Y., Chen, C.: Biosynthesis of silver and gold nanoparticles by novel sundried Cinnamomum camphora leaf. Nanotech. 18, 105104 (2007)

14. Alam, M.N., Roy, N., Mandal, D., Begum, N.A.: Green chemistry for nanochemistry: exploring medicinal plants for the biogenic synthesis of metal NPs with fine-tuned properties. RSC Adv. 3, 11935-11956 (2013)

15. Begum, N.A., Mondal, S., Basu, S., Laskar, R.A., Mandal, D.: Biogenic synthesis of $\mathrm{Au}$ and $\mathrm{Ag}$ nanoparticles using aqueous solutions of black tea leaf extracts. Colloids Surf. B Biointerf. 71, 113 (2009)

16. Alam, MdN, Das, S., Batuta, S., Roy, N., Chatterjee, A., Mandal, D., Begum, N.A.: Murraya koenegii Spreng. leaf extract: an efficient green multifunctional agent for the controlled synthesis of Au nanoparticles. ACS Sustain. Chem. Eng. 2, 652-664 (2014)

17. Alam, M.N., Batuta, S., Ahamed, G., Das, S., Mandal, D., Begum, N.A.: Tailoring the catalytic activity of Au nanoparticles synthesized by a naturally occurring green multifunctional agent. Arab. J. Chem. (2016). doi:10.1016/j.arabjc.2016.02.007

18. Kumar, B., Yadav, P.R., Goel, H.C., Rizvi, M.M.A.: Recent developments in cancer therapy by the use of nanotechnology. Digest J. Nanomat. Biostruct. 4, 1-12 (2009)

19. Aragay, G., Pino, F., Merkoci, A.: Nanomaterials for sensing and destroying pesticides. Chem. Rev. 112, 5317-5338 (2012)

20. Rohit, J.V., Solanki, J.N., Kailasa, S.K.: Surface modification of silver nanoparticles with dopamine dithiocarbamate for selective colorimetric sensing of mancozeb in environmental samples. Sens. Actuators B. 200, 219-226 (2014)

21. Xiong, D., Li, H.: Colorimetric detection of pesticides based on calixarene modified silver nanoparticles in water. Nanotech. 19, 465502-465507 (2008)

22. Menon, S.K., Modi, N.R., Pandya, A., Lodha, A.A.: Ultrasensitive and specific detection of dimethoate using p-sulphonato calix [4] resorcinarene functionalized silver nanoprobe in aqueous solution. RSC Adv. 3, 10623-10627 (2013)

23. Jain, V., Momin, M., Laddha, K.: Murraya koenigii: an updated review. Int J Ayurvedic Herb Med 2, 607-627 (2012)

24. Singh, A.P., Wilson, T., Luthria, D., Freeman, M.R., Scott, R.M., Bilenker, D., Shah, S., Somasundaram, S., Vorsa, N.: LCMS-MS characterisation of curry leaf flavonols and antioxidant activity. Food Chem. 127, 80-85 (2011)

25. Chang, C., Yang, M., Wen, H., Chern, J.: Estimation of total flavonoids content in propolis by two complementary colorimetric methods. J. Food Drug Anal. 10, 178-182 (2002)

26. Mcdonald, S., Prenzler, P.D., Autolovich, M., Robards, K.: Phenolic content and antioxidant activity of olive extracts. Food Chem. 73, 73-84 (2001)

27. Roy, N., Alam, M.N., Mondal, S., Sk, I., Laskar, R.A., Das, S., Mandal, D., Begum, N.A.: Exploring Indian Rosewood as a promising biogenic tool for the synthesis of metal nanoparticles with tailor-made morphologies. Process Biochem. 47, 1371-1380 (2012)

28. Link, S., Wang, Z.L., El-Sayed, M.A.: Alloy formation of goldsilver nanoparticles and the dependence of the plasmon absorption on their composition. J. Phys. Chem. B 103, 3529-3533 (1999)

29. Ahmad, A., Mukherjee, P., Senapati, S., Mandal, D., Khan, M.I., Kumar, R., Sastry, M.: Extracellular biosynthesis of silver nanoparticles using the fungus Fusarium oxysporum. Coll. Surf. B Biointerfaces. 28, 313-318 (2003)

30. Shankar, S.S., Ahmad, A., Khan, A.M.I., Sastry, M., Kumar, R.: Extracellular biosynthesis of bimetallic $\mathrm{Au}-\mathrm{Ag}$ alloy nanoparticles. Small 1, 517-520 (2005) 
31. Begum, N.A., Roy, N., Mandal, S., Basu, S., Mandal, D.: Fluorescence spectroscopy of a naturally occurring carbazole alkaloid: murrayanine. J. Lumin. 129, 158-163 (2009)

32. Senkbeil, S., Lafleur, J.P., Jensen, T.G., Kutter, J.P.: Gold nanoparticle-based fluorescent sensor for the analysis of dithiocarbamate pesticides in water. 16th international conference on miniaturized systems for chemistry and life sciences, Oct 28Nov 1, Okinawa, Japan, pp 1423-1425 (2012) 